\title{
Yak rumen microbial diversity at different forage growth stages of an alpine meadow on the Qinghai-Tibet Plateau
}

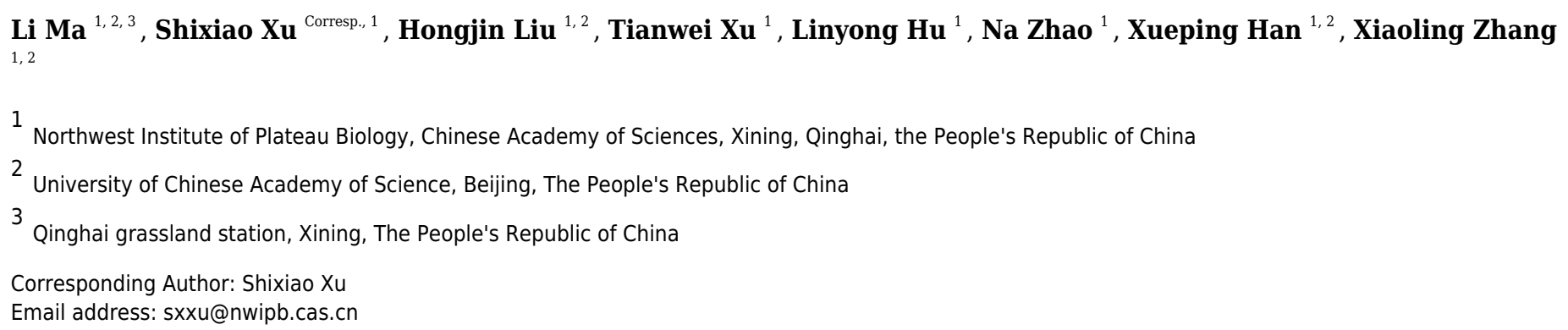

The rumen microbiota of ruminants plays a vital role in fiber digestion, and environmental factors affect its community structure. The yak (Bos grunniens) is the main livestock species that inhabits the Qinghai-Tibet Plateau (QTP) at regions located at high-altitude of 3000-5000 m. This work investigated the rumen bacterial community of yak that grazed on the QTP during the whole year to evaluate the relationship between the rumen bacterial community and the nutrient composition of forage plant at three stages. In this study, the diversity of the rumen prokaryotic community composition was monitored in 10 fullgrazing yak in an alpine meadow of the QTP. The nutrient composition of three forage growth stages was determined: re-green stage (REGY), grassy stage (GY), and withered stage (WGY). High-throughput sequencing of bacterial 16S rRNA gene was used. The results showed that the nutritive composition of the alpine meadow changed with the seasons: crude protein (CP) (13.22\%) was high in forage during REGY (spring), while neutral detergent fiber (NDF) (59.00\%) was high during WGY (winter). Microbial diversity and richness were highest during REGY and the average number of operational taxonomic units from 30 samples was 4470 . The microbial composition was dominated by members of Bacteroidetes (51.82\%), followed by Firmicutes (34.08\%), and the relative microbial abundance changed in the three forage growth stages. Unweighted UniFrac distance PcoA showed that the bacterial community structure differed between REGY, GY, and WGY. Furthermore, taxonomic groups did not present differences regarding gender in these three stages. The rumen microbiota was enriched with functional potentials that were related to $A B C$ transporters, the Two component system, Aminoacyl-tRNA biosynthesis, and metabolism of Purine, Pyrimidine, Starch and sucrose metabolism. Significant differences were found in the composition, diversity, and function of yak ruminal microorganisms during different forage growth stages. This indicates that microbial changes in the rumen denend on changes in the forage nutritional composition. These
Peerf reviewing PDF | (2018:11:32825:3:0:ACCEPTED 5 Aug 2019) 
findings provide evidence on the rumen microbial diversity of yaks in the QTP. 
1 Yak rumen microbial diversity at different forage growth stages of an alpine

\section{2 meadow on the Qinghai-Tibet Plateau}

3

$4 \mathrm{Li} \mathrm{Ma}^{1,2,3}$, Shixiao Xu, Corresp., ${ }^{1}$, Hongjin Liu ${ }^{1,2}$, Tianwei Xu ${ }^{1}$, Linyong $\mathrm{Hu}{ }^{1}$,

5 Na Zhao ${ }^{1}$, Xueping Han ${ }^{1,2}$, Xiaoling Zhang ${ }^{1,2}$

6

71 Northwest Institute of Plateau Biology, Chinese Academy of Sciences, Xining,

8 Qinghai, the People's Republic of China

92 University of Chinese Academy of Science, Beijing, The People's Republic of

10 China

113 Qinghai grassland station, Xining, The People's Republic of China

13 Author:Li Ma. Email address: 942845751@qq.com

15 Corresponding Author: Shixiao Xu, Doctor, research direction: Animal Ecology,

16 Email address: sxxu@nwipb.cas.cn 
18 Yak rumen microbial diversity at different forage growth stages of an alpine

\section{meadow on the Qinghai-Tibet Plateau}

\section{Abstract}

The rumen microbiota of ruminants plays a vital role in fiber digestion, and environmental factors affect its community structure. The yak (Bos grunniens) is the main livestock species that inhabits the QinghaiTibet Plateau (QTP) at regions located at high-altitude of 3000-5000 m. This work investigated the rumen bacterial community of yak that grazed on the QTP during the whole year to evaluate the relationship between the rumen bacterial community and the nutrient composition of forage plant at three stages. In this study, the diversity of the rumen prokaryotic community composition was monitored in 10 fullgrazing yak in an alpine meadow of the QTP. The nutrient composition of three forage growth stages was determined: re-green stage (REGY), grassy stage (GY), and withered stage (WGY). High-throughput sequencing of bacterial 16S rRNA gene was used. The results showed that the nutritive composition of the alpine meadow changed with the seasons: crude protein (CP) (13.22\%) was high in forage during REGY (spring), while neutral detergent fiber (NDF) (59.00\%) was high during WGY (winter). Microbial diversity and richness were highest during REGY and the average number of operational taxonomic units from 30 samples was 4470 . The microbial composition was dominated by members of Bacteroidetes (51.82\%), followed by Firmicutes (34.08\%), and the relative microbial abundance changed in the three

forage growth stages. Unweighted UniFrac distance PcoA showed that the bacterial community structure differed between REGY, GY, and WGY. Furthermore, taxonomic groups did not present differences regarding gender in these three stages. The rumen microbiota was enriched with functional potentials that were related to $\mathrm{ABC}$ transporters, the Two component system, Aminoacyl-tRNA biosynthesis, and metabolism of Purine, Pyrimidine, Starch and sucrose metabolism. Significant differences were found in the composition, diversity, and function of yak ruminal microorganisms during different forage growth stages. This indicates that microbial changes in the rumen depend on changes in the forage nutritional composition. These findings provide evidence on the rumen microbial diversity of yaks in the QTP.

\section{Introduction}

The yak (Bos grunniens) is the most important animal species in the QTP, China, which is the most 
45

46

47

important terrestrial ecosystem in Eurasia (Harris 2010). The yak is adapted to the harsh environment of the QTP that possesses low oxygen, strong ultra-violet (UV) radiation, and limited forage resources. Natural alpine meadows in the QTP also have qualities of low temperature, high altitude, high variability in temperature and precipitation, and these factors directly affect plant productivity and nutrition (Xue et al. 2005; Huang et al. 2017). To the growth period of grass is approximately from 100 to $150 \mathrm{~d}$ per year, and the dormant period lasts for about 7 months. Grasses begin to regreen each year in April, reach their peak biomass in August, and wither in November. Yaks are kept at a full-grazing style with coarse grasses as the only food in the QTP, which leads to the particular phenomenon the local herdsmen call the yak production cycle (satiate in summer, fatten in fall, thin in winter, and die in spring) (Long et al. 1999). To avoid the death of yaks in spring and their decrease in body weight (BW) over winter, supplementary feeding with total mixed rations of available roughage and grains is widely used as the grazing system (Xue et al. 2017).

It has been reported that the yak has a special molecular mechanisms to adapt to the harsh living environment in the cold and low-oxygen environment (Chen et al. 2015a; Xue et al. 2017) and yaks have adapted to the poor forage resources of the QTP. The rumen as the first chamber of the ruminant stomach, is populated by bacteria, fungi, archaea (methanogens), and protozoa with important functions in the digestion of the complex cellulolytic biomass. Ruminal microorganisms synergistically ferment plants and then provide nutrients to the host in the form of short-chain fatty acids and microbial proteins (Huang et al. 2017). To enhance the fiber digestibility and feed utilization, it is necessary to obtain a deep understanding of the microorganisms in the rumen. Firmicutes and uncultured species were reported to have higher abundance in the yak of the QTP compared to yak at low elevation, and several bacteria (Ruminococcus albus and Prevotella ruminicola) were either not detected or had lower abundance among fibrolytic bacteria (Ruminococcus, Fibrobacter, Clostridium, and Butyrivibrio) in the yaks of the QTP (Yang et al. 2010; Dan et al. 2016; Huang et al. 2017; Xue et al. 2017; Xue et al. 2018). Previous studies have shown that factors such as diet, age, species, and seasons all impact on ruminal microbes, and diet is a particularly important contributor (Tajima et al. 2000). It has also been suggested that unique bacterial communities are present in ruminants of the QTP (Chen et al. 2015b; Huang et al. 2017). Xue et al. 
(2017) reported the rumen bacteria and compared the differences of microorganisms between barn fed and grazing yak in the QTP; however, these grazed yak were only studied in October, when forage biomass and nutrients peaked and did not consider the cold season. Diet and environmental conditions influence the microbial community composition; however, little is known about the bacterial community changes over time when ruminants are on similar diets, especially in the QTP where the forage differs considerably throughout a whole year due to the harshness of the environment. Some studies have been conducted on rumen microbiota in grazing yaks and sheep in QTP (Dan et al. 2016; Huang et al. 2017; Guo et al. 2018). Changes in temperature and precipitation directly affect plant yak productivity, which in turn affects animal growth and the microbiome in the body. Bacteroidetes and Firmicutes were the two predominant in the rumen of yak, these two phyla accounting for approximately $80 \%$ of the total reads, the remaining microbes involved in Fibrobacter, Spirochaeta, and Proteobacteria consisted of lowabundance phyla ( $<10 \%$ of the total reads). At the genus level, Prevotella, Butyrivibrio, Fibrobacter, RC9_gut_group and BS11_gut_group_norank and the unclassified bacteria were identified as the dominant genera in the rumen bacterial community (Chen et al. 2015b; Peng et al. 2015; Huang et al. 2017).

Ruminal microbial composition has first been described using traditional culture-based methods (Dehority et al. 1989), which was followed by molecular studies (Morozumi et al. 2006; FernandezGuerra et al. 2010; Sadet-Bourgeteau et al. 2010; Bekele et al. 2011; Klitgaard et al. 2013) in recent years. The rumen bacterial diversity has been substantially underestimated by traditional methods due to its anaerobiosis, which is difficult to study outside of the animal's rumen and molecular techniques based on the amplification of $16 \mathrm{~S} / 18 \mathrm{~S}$ rRNA gene fragments were widely used for the study of rumen microbes. High-throughput sequencing technology has provided microbial compositions of a wide variety of different ecosystems as well as provided biological information of many microorganisms without the need for prior cultivation. Here, high-throughput sequencing of the V3-V4 region of 16S rRNA gene was used to study yak rumen bacterial community among different forage growth stages and between female and make yaks in the QTP. This study aimed to compared the composition, diversity and functions of rumen microbiota of yak under different forage growth stages and between female and make yaks. We 
99 hypothesized that documenting concurrent rumen microbiota shifts in different forage growth stage will

help us establish variation trend in yak rumen community composition throughout the year. This study greatly enhanced our understanding of changes in diet corresponding shifts in rumen microbial community composition throughout the year. The knowledge of yak microbial communities in different forage growth stage can promote the understanding of rumen microbial ecosystems and improve yak productivity.

\section{Materials and methods}

The experimental design and procedures were approved by the Northwest Institute of Plateau Biology, CAS-Institutional Animal Care and Use Committee (NWIPB20160302), besides this project was carried out with the permission of the local government (Qinghai province, China), so official documents and field permits are not required in this study, the China Qinghai provincial science \& technology department gave these authorizations to granting us to carry out the experiment here.

\section{Experimental design}

In the montane grassland of the Senduo Township, Guinan County, Hainan Prefecture, Qinghai Province, China, the elevation of the studied sample plot was $3265 \mathrm{~m}$. The sample plot had a latitude of $35^{\circ} 31^{\prime} \mathrm{N}$ and a longitude of $100^{\circ} 55^{\prime} \mathrm{E}$; the annual average temperature is $1.3{ }^{\circ} \mathrm{C}$ with extended daylight times and strong solar radiation. The abundance of solar energy is evidenced by the number of annual sunshine hours of $1252-1333 \mathrm{~h}$ and the forage grass growth period is $160-171 \mathrm{~d}$. The cumulative $\geq 0{ }^{\circ} \mathrm{C}$ temperature is $1587.4{ }^{\circ} \mathrm{C}$ and the experimentally studied duration was 9 months (April 2017 to December 2017). Data for long-term mean climate variables as obtained from the Qinghai meteorological station. Ten healthy three-year-old QTP yaks (five males and five females) with an average weight of $108.06 \pm$ $2.86 \mathrm{~kg}$ were selected, sequentially numbered, and allowed to graze. These ten yaks, without supplementary feeding during the experimental stage, were allowed access to water. Ruminal fluid was collected during the following forage growth stage: re-greening grass growth stage (REGY, spring), grass growth stage (GY, summer), and withered grass growth stage (WGY, winter), and alpine meadow samples were collected during the same three stages. The main forage species and their relative proportions in montane grassland during these three periods are listed in Table 1. Biomass was highest in 
126

127

128

129

130

131

132

133

134

135

136

137

138

139

140

141

142

143

144

145

146

147

148

149

150

151

152

July at $146.56 \mathrm{~g} / \mathrm{m}^{2}$ and the ratio of high-quality forage to the total above-ground biomass was $55-61 \%$. The proportion of dominant species of the forage were Elymus nutansv (74.5\%), Kobresia humili (6.8 \%), and Poa pratensiv (2.0\%), while further species include Potentill abifurca (3.4\%), Saussurea pulchra (2.7 \%), and Ajania tenuifolia (1.4\%) in GY. The proportion of dominant species of the alpine meadow in REGY were Gramineae (40.9\%), Cyperaceae (36\%), and Potentilla (2.04\%) plants, and the dominant grass species was Elymus nutans (78.56\%) in WGY (Table S1).

\section{Plant sample collection}

Forage samples were collected by quadrats $(1 \mathrm{~m} \times 1 \mathrm{~m})$ from grass on which the animals grazed during the regreen stage (May 2, 2017), the grass stage (July 12, 2017), and the withered grass stage (December 7, 2017). Ten quadrats $(1 \mathrm{~m} \times 1 \mathrm{~m})$, of which the distance between plots exceeded $10 \mathrm{~m}$, were randomly placed in the alpine meadow to collect grass samples and cut out the ground part of the quadrats with scissors at three sample collection times. Samples from each quadrat were used to investigate dominant species, dried in a $60{ }^{\circ} \mathrm{C}$ oven for $24 \mathrm{~h}$ to constant weight at the laboratory, and were ground in a mill and passed through a 1-mm sieve for further analysis.

\section{Rumen fluid collection}

The rumen contents (30 samples) were collected using an esophageal tube vacuum pump sampling device (Anscitech Company, Wuhan, China). This method has been used in previous studies (RamosMorales et al. 2014; Xue et al. 2017; Sun et al. 2018), and they have shown that the stomach tube and rumen cannulation methods do not have any influence on the results. The rumen contents were filtered using four layers of gauze and approximately $75 \mathrm{~mL}$ of fluid was collected from each animal prior to grazing in the morning. Twenty $\mathrm{mL}$ of fluid was aliquoted for use and $2 \mathrm{~mL}$ was used for DNA extraction and subsequent sequencing. After aliquoting, the fluid samples were immediately placed into liquid nitrogen $\left(-80^{\circ} \mathrm{C}\right)$ for storage and transported to the laboratory for further use.

\section{Determination of plant nutrient compositions}

The concentration of acid detergent fiber (ADF), and neutral detergent fiber (NDF) in 30 samples (10 samples per period) were determined using the Van Soest method (Van Soest et al. 1991). Dry matter $(\mathrm{DM})$, crude protein $(\mathrm{CP})$, crude fat ether extract $(\mathrm{EE}), \mathrm{Ca}$, and $\mathrm{P}$ were measured using AOAC methods 
153

154

155

156

157

158

159

160

161

162

163

164

165

166

167

168

169

170

171

172

173

174

175

176

177

178

179

(Cunniff 1995).

\section{DNA extraction and polymerase chain reaction (PCR) amplification}

Microbial DNA was extracted from samples using the E.Z.N.A. stool DNA Kit (Omega Biotek, Norcross, GA, USA) according to the manufacturer's instructions. The 16S rDNA V3-V4 region of the Prokaryotic ribosomal RNA gene was amplified by PCR $\left(95^{\circ} \mathrm{C}\right.$ for $2 \mathrm{~min}$, followed by 27 cycles at $98{ }^{\circ} \mathrm{C}$ for $10 \mathrm{~s}, 62{ }^{\circ} \mathrm{C}$ for $30 \mathrm{~s}, 68^{\circ} \mathrm{C}$ for $30 \mathrm{~s}$, and a final extension at $68^{\circ} \mathrm{C}$ for 10 min using primers $341 \mathrm{~F}$ : CCTACGGGNGGCWGCAG; 806R: GGACTACHVGGGTATCTAAT (Guo et al. 2017). Amplicons were extracted from 2\% agarose gels and purified using the AxyPrep DNA Gel Extraction Kit (Axygen Biosciences, Union City, CA, USA) according to the manufacturer's instructions and quantified using QuantiFluor-ST (Promega, USA). According to the standard protocols, Purified amplicons were sequenced on an Illumina Hiseq2500 platform (Illumina San Diego, CA, USA), which was conducted by Genedenovo Bioinformatics Technology Co., Ltd. (Guangzhou, China) using 250bp paired end reads.

\section{Data analysis}

Sequences were sorted based on their unique barcode and then, barcode and primer sequence were removed using QIIME (version 1.9.0). FLSAH (version 1.2.11) was used to merge paired end clean reads as raw tags (Magoc \& Salzberg, 2011). Low quality reads were eliminated to obtain high-quality clean tags by QIIME (version 1.9.0) (Caporaso et al. 2010). Clean tags were compared to the Gold database (http://drive5.com/uchime/uchime_download.html) using the UCHIME algorithm

(http://www.drive5.com/usearch/manual/uchime_algo.html) to eliminate chimera sequences and effective tags were obtained for further analysis. These effective tags were clustered into operational taxonomic units (OTUs) of $\geq 97 \%$ similarity using the UPARSE pipeline (Edgar 2013). Representative sequences were classified into organisms using the RDP classifier (Wang et al. 2007) based on the SILVA database (Quast et al. 2013).

\section{Statistical analysis}

Alpha diversity analysis including Chao1, Shannon, Simpson, ACE, and Good-coverage were calculated by QIIME (version 1.9.0). Alpha index comparison among groups was computed by KruskalWallis test in R (version 3.4.3). A principal coordinate analysis (PCoA) and ANOSIM were used to 
confirm findings from the distance matrices. Pearson correlations were conducted using R software (version 3.4.3) with the vegan package on the online omicsmart cloud platform (http://www.omicsmart.com/). Pearson correlations using the pheatmap package were used to analyze the relationships between environmental factors and bacteria.

Relative differential abundance tests among REGY, GY, and WGY were also conducted at phylum, genus, and family levels using Kruskal-Wallis test in R. Tukey-Kramer post-hoc test was used in conjunction with analysis of Student's t-test in STAMP (version 2.1.3) to compare the abundance changes between female and male yaks. Pearson correlations in the omicsmart cloud platform was used to examine the relationships among the 20 known prokaryotic genera with six forage nutrient compositions, redundancy analysis in Canoco (version 5.0) was used to examine relationships of the top 10 known prokaryotic phyla with six forage nutrient compositions. Nutrient compositions of the forage were analyzed using a general linear model, and a calculated $\mathrm{P}$ value $<0.05$ was considered to indicate statistical significance. The differences between Diversity and richness mean values were analysed using two-way anova test conducted with SAS 9.4 software (SAS Inst. Inc., Cary, NC, USA). Tax4fun software was used to compare the species compositions obtained from the 16S sequencing data and then to infer the functional gene composition of samples. The functional composition was predicted from the Kyoto Encyclopedia of Genes and Genomes (KEGG) database (Minoru et al. 2012). The KOs (KEGG orthology groups) at different forage growth stages were further examined by Kruskal-Wallis test in R.

\section{Accession number}

The sequencing data for the $16 \mathrm{~S}$ rRNA genes are publicly available in the NCBI (https://www.ncbi.nlm.nih.gov/) Short Read Archive under accession. No. PRJNA504932.

\section{Results and analysis}

\subsection{Analysis of forage nutrient composition}

The nutrient composition of mixed forage samples collected during different forage growth stages from the QTP was determined (Table 1). The CP content was the highest during REGY and was significantly $(\mathrm{P}<0.05)$ higher than during WGY, the REGY presented $1.0 \%$ more protein than GY. In GY, the EE content was highest and was significantly $(\mathrm{P}<0.05)$ higher than during REGY, the GY was 
207

208

209

210

211

212

213

214

215

216

217

218

219

220

221

222

223

224

225

226

227

228

229

230

231

232

233

$0.43 \%$ higher than REGY. ADF reached $35.53 \%$ in REGY that higher than other two stages. NDF gradually increased in the trend: REGY $<$ GY $<$ WGY, the NDF content was highest in WGY and was significantly $(\mathrm{P}<0.05)$ higher than REGY. Ca and $\mathrm{P}$ were highest during $\mathrm{GY}$ and the Ca content was significantly higher during GY $(\mathrm{P}<0.05)$ compared to REGY and WGY. The main dominant species during each grazing period are shown in Table 1. The nutrient composition of herbage varies in 3 forage growth stages. The nutritive value and palatability of herbage in the REGY was higher than WGY, that is, the CP content was increased with NDF decline in REGY, whereas the NDF content was increased with CP decline in WGY.

\subsection{Sequencing results and diversity analysis of forage samples}

By sequencing the V3-V4 of the 16S rRNA gene of rumen bacteria collected during the three forage growth stages, 3,104,385 raw sequences were obtained from 30 samples. After filtering low-quality sequences, the total number of effective sequences was 3,059,694. All effective tags of 30 samples were clustered into 13,280 OTUs at the similarity $>97 \%$, averaged at 4,447 OTUs per sample. There were 7,064 OTUs shared among REGY, GY, and WGY (Fig. 1, Table S2); these 7,064 OTUs accounted for 53\% of all 13,280 OTUs and Bacteroidetes, Firmicutes, Verrucomicrobia, and Proteobacteria accounted for $23.56 \%, 17.90 \%, 3.28 \%$, and $2.44 \%$ of all 13,280 OTUs, respectively. The diversity and richness indices of all samples from the three groups and genders were calculated. The number of species observed in the rumen fluid was significantly different among three grass growth stage $(p-$ value $=0.03)$, with the highest number in female samples from REGY. Index ACE observed in the rumen fluid was significantly different among three grass growth stage $(p-$ value $=0.02)$, with the highest number in female samples from REGY (Table 2).

\subsection{Composition of rumen bacterial community}

At the phylum level, the top ten phyla were accounted for nearly $99 \%$ of all sequences. The average abundance of the three groups in 30 samples were Bacteroidetes (51.82\%), Firmicutes $(34.08 \%)$, Verrucomicrobia (5.97\%), Proteobacteria (1.92\%), Spirochaetae (1.12\%), Lentisphaerae (1.07\%), Tenericutes (0.93\%), SR1 (0.92\%), Cyanobacteria (0.62\%), and Fibrobacteres (0.35\%) (Table. S2).

Firmicutes had the highest relative abundance during GY, while Verrucomicrobia, Lentisphaerae, 
234 Cyanobacteria, and Fibrobacteres gradually increased in the trend, following: GY $<$ REGY $<$ WGY. 235 Among the top 10 phyla, Kruskal-Wallis test demonstrated the abundance of Firmicutes ( $p$ - value $<$ 236 0.01), Verrucomicrobia ( $p$ - value $<0.01)$, Proteobacteria $(p$ - value $<0.01)$, Lentisphaerae $(p$ - value $<$ 237 0.01), and Fibrobacteres ( $\mathrm{P}<0.01$ ) changed significantly at REGY, GY, and WGY, At the phylum level, 238 Firmicutes was significantly more abundant in GY than in REGY ( $p$ - value $<0.05)$ or WGY ( $p$ - value $<$ 239 0.05). Verrucomicrobia was significantly lower in GY than in REGY ( $p$ - value $<0.01)$ and WGY $(p-$ 240 value $<0.01)$, Proteobacteria was significantly lower in REGY than in GY ( $p$ - value $<0.01)$ or WGY ( $p$ 241 - value <0.01), Lentisphaerae was significantly higher in WGY than in REGY $(p$ - value < 0.01) or GY $242(p-$ value $<0.01)$ (Fig. 2).

243 At the class level, Clostridia (p_Firmicutes), Negativicutes (p_Firmicutes), and Erysipelotrichia 244 ( $p \_$Firmicutes) all were significantly more abundant in GY than in REGY or WGY just as Firmicutes. 245 Deltaproteobacteria ( $p \_$Proteobacteria) was significantly more abundant in GY than in REGY ( $p$ - value $246=0.006)$ or WGY ( $p$ - value < 0.013) (Fig. S1A). At the order level Victivallales (c_Lentisphaeria, 247 p_Lentisphaerae), Oligosphaerales (c_Oligosphaeria, p_Lentisphaerae), Gastranaerophilales 248 (c_Melainabacteria, $p_{-}$Cyanobacteria) were significantly higher in WGY than in REGY and GY (Fig. 249 S1B).

At the family level, the top 10 families accounted for $81.04 \%$ of the total sequences (Fig. 3, Table S3). Among these, family Prevotellaceae (p_Bacteroidetes) (REGY: 24.76\%, GY: $21.37 \%$, WGY: 26.09\%) was the most dominant family, followed by Ruminococcaceae (p_Firmicutes) (REGY: 14.19\%, GY: 16.50\%, WGY: 13.56\%), Rikenellaceae (p_Bacteroidetes) (REGY: 11.59\%, GY: 11.44\%, WGY: 11.96\%), and Lachnospiraceae (p_Lentisphaerae) (REGY: 12.62\%, GY: 12.06\%, WGY: 8.50\%). Other families with lower abundance were Bacteroidales_BS11_gut_group (REGY: 9.85\%, GY: 9.10\%, WGY: 7.39\%), Bacteroidales_S24-7_group (REGY: 2.64\%, GY: 5.00\%, WGY: 2.67\%), Christensenellaceae (p_Firmicutes) (REGY: 2.86\%, GY: 4.95\%, WGY: 1.97\%), Veillonellaceae (p_Firmicutes) (REGY: 1.58\%, GY: 3.12\%, WGY: 0.45\%), Bacteroidales_RF16_group (REGY: 1.33\%, GY: 1.17\%, WGY: 1.69\%), and Family_XIII (p_Firmicutes) (REGY: 0.74\%, GY: 1.24\%, WGY: 0.69\%). Kruskal-Wallis test 260 demonstrated that the abundances of Ruminococcaceae ( $p$ - value $<0.05)$, Lachnospiraceae ( $p$ - value $<$ 
261

262

263

264

265

266

267

268

269

270

271

272

273

274

275

276

277

278

279

280

281

282

283

284

285

286

287

0.01), Bacteroidales_S24 -7_group ( $p$ - value $<0.05)$, Christensenellaceae $(p$ - value $<0.01)$, Veillonellaceae $(p$ - value $<0.01)$, and Family_XIII $(p$ - value $<0.01)$ changed significantly at all three groups (Fig. 3). At the family level, Ruminococcaceae, Bacteroidales_S24-7_group, Christensenellaceae, Veillonellaceae, and Family_XIII all were significantly higher in GY than in REGY. Lachnospiraceae was significantly lower in WGY than in REGY $(p$ - value $<0.01)$ or GY $(p$ - value $<0.01)$.

At the genus level, taxa with a relative abundance of $\geq 1 \%$ in at least one sample were analyzed.

Sixteen taxa exhibited significantly different abundances between three groups were particularly dominant, including Christensenellaceae_R-7_group $(p$ - value $<0.01)$, Prevotellaceae_UCG-003 ( $p$ value $<0.01)$, Prevotellaceae_UCG-001 ( $p$ - value $<0.05)$, Butyrivibrio_2 $(p$ - value $<0.01)$, Ruminococcaceae_UCG-010 ( $p$ - value $<0.01)$, Ruminococcaceae_UCG-005 ( $p$ - value $<0.01)$, Ruminococcaceae_UCG-014 ( $p$ - value $<0.01)$,Ruminococcaceae_NK4A214_group $(p$ - value $<0.01)$, Selenomonas_1 (f_Veillonellaceae), Ruminococcus_1 (f_Ruminococcaceae), Saccharofermentans (f_Ruminococcaceae), and Pseudobutyrivibrio (f_Lachnospiraceae). Prevotella_1 (15.76\%), and Rikenellaceae_RC9_gut_group (11.17\%) (Table S3). At the genus level, Christensenellaceae_R-7_group, Butyrivibrio_2, Ruminococcaceae_UCG-005, Ruminococcaceae_NK4A214_group all were significantly $(p$ - value $<0.01$ ) more abundant in GY than in REGY or WGY. Prevotellaceae_UCG-003 and Prevotellaceae_UCG-001 were lowest abundance in the GY compared with other two groups (Fig. 4, Table S3).

Furthermore, Student's t-test was used to assess significant differences in species abundance between female and male yak populations at three groups at phylum, family, and genus levels (Fig. 5). Two phyla exhibited significantly different abundances between female and male yaks, Tenericutes (REGY) was more abundant in the female group at ( $p$ - value $<0.05$ ), and Bacteroidetes (WGY) was more abundant in the male group at $\mathrm{P}<0.05$. Two families including Bacteroidales_S24-7_group and Family_XIII were more abundant in the female yak group during GY $(p$ - value $<0.05)$, and Prevotellaceae were more abundant in the male yak group during WGY ( $p$ - value $<0.01)$. Moreover, two genera including Prevotella_1 (WGY_female: 15.07\%, WGY_male: 18.99\%) and Rikenllaceae_RC9_gut_group (GY_female: 12.02\%,GY_male: 9.68\%) exhibited significantly different 
288

289

290

291

292

293

294

295

296

297

298

299

300

301

302

303

304

305

306

307

308

309

310

311

312

313

314

abundances between female and male yak groups ( $p$ - value $<0.05)$.

3.4 Microbial community analysis at three forage growth stages

PCoA was used to compare the bacterial community structure among different forage growth stages (Fig. 6). Unweighted UniFrac distance PcoA showed that the GY group community structure differed significantly from that of the other two groups (ANOSIM: Unweighted unifrac, $p$-value $=0.001, R$-value $=0.662$ ). Significant differences (based on ANOSIM tests) were also found between REGY and WGY groups $(p$-value $=0.001, R$-value $=0.282)$. It was not detected differences in PcoA between female and male samples.

\subsection{Correlations of microbial communities with forage nutrient composition}

To study the relationships of the bacterial community structure with forage nutrient parameters, correlations were investigated using both Pearson correlations and redundancy analysis. Fig. 7A shows that CP correlated positively with relative abundance of Christensenellaceae_R-7_group, Butyrivibrio_2, Ruminococcaceae_UCG-005, Ruminococcaceae_UCG-014,Ruminococcaceae_NK4A214_group, Selenomonas_1, and Pseudobutyrivibrio $(r$-value $>0.4, p$-value $<0.05)$, but correlated negatively with the Coprostanoligenes_group $(r$ - value $<-0.4, p$ - value $<0.05)$. NDF correlated negatively with the relative abundances of Ruminococus_1, Saccharofermentans, Prevotellaceae_UCG-003, and Ruminococcaceae_UCG-014 ( $r$ - value $<-0.4, p$ - value $<0.05)$. Ca correlated positively with the relative abundances of Ruminococcaceae_UCG-005 and Pseudobutyrivibrio ( $r$-value $>0.4, p$ - value $<0.05$ ), but negatively with relative abundances of Ruminococcaceae_UCG-003, Ruminococcus_1, and Saccharofermentans $(r$ - value $<-0.4, p$ - value $<0.05)$ (Table S4).

Redundancy analysis indicated the correlation between the bacterial community structure at the phylum level and forage nutrition parameters, the two axes explained $65.07 \%$ of the differentiation of the microbial community. CP, P, Ca, NDF, and EE all correlated positively with Firmicutes, however correlated negatively with Bacteroidetes. Proteobacteria correlated positively with NDF and EE, Spirochaetae correlated positively with NDF, Ca, and EE (Fig. 7B), Verrucomicrobia, Lentisphaerae, and Fibrobacteres all correlated negatively with CP.

\subsection{Tax4fun gene function prediction}


Yak rumen liquid bacterial community functional predictions in the three forage growth stages were investigated using Tax4fun4 and differences in KEGG and KO abundances between groups were identified. KOs in level 2 (Table S5) suggest that the pathways related to membrane transport, translation, and carbohydrate metabolism were enriched in REGY, GY, and WGY groups. At the pathway level, compared to GY group (Level 2 KOs, student's-t test, $\mathrm{p}<0.05$, Fig. 8), genes involved in Cell growth and death, Cell motility, Membrane transport, and Carbohydrate metabolism were significantly more abundant in the REGY group, while genes that included Folding, sorting, and degradation, Cell growth and death, Translation, Glycan biosynthesis, and Metabolism and energy metabolism were significantly lower than the WGY $(p$ - value $<0.05)$. The relative abundance of Metabolism of cofactors and vitamins was significantly higher in GY than in WGYS groups ( $p$ - value $<0.05$ ).

The relative abundance of $\mathrm{ABC}$ transporters (7.138 \%) was highest in the three stages, AminoacyltRNA biosynthesis (3.66\%), Starch and sucrose metabolism (2.66\%), Ribosome (2.32\%) as well as Amino Sugar and nucleotide sugar metabolism (2.22\%) were second-most abundant KOs. Kruskal-Wallis test demonstrated that a total of 181 KOs showed significant difference at REGY, GY, and WGY groups $(p$ - value $<0.05)$ in the level three KEGG pathways, 23 KOs (relative abundances $>1 \%$ ) of which are shown in Fig. 9. The heatmap (Fig. S2) also indicated that the relative abundance of Fructose and mannose metabolism, Alanine, aspartate and Glutamate metabolism, and Cysteine and methionine metabolism in WGY group were higher than in the other two groups.

\section{Discussion}

According to prior analyses (CHEN et al. 2008; Latham et al. 2018), low-quality diets in low protein supplementation had greater bacterial diversity, which could be interpreted as a further feature of the diet that contains more secondary plant compounds in low- quality diets compared to high- quality diets. (Belanche et al. 2012; Fernandes et al. 2014) also reported that a fiber-based diet promotes higher levels of dietary microbial diversity since fiber fermentation produces more by-products compared to starch fermentation. This is consistent with the results of this study where forage nutrient quality decreased, while microbial diversity increased in WGY compared to GY and REGY. Compared to other factors such 
342 as the environment, rumen microbial diversity is strongly affected by diet. The results of this study show 343 that during different forage growth stage, bacterial composition remains basically identical, however, the 344 relative abundance of microbes differs. Dan et al. (2016) reported that the yak grazing on the QTP, 345 Bacteroidetes and Firmicutes were the two predominant bacteria, representing $59.06 \%$ and $25.81 \%$ of all 346 sequence, respectively. The same conclusion was obtained in the present study where the relative 347 abundances of these two phyla reached $51.84 \%$ and $34.16 \%$. The similar proportion in relative abundance 348 of these two phyla may be attributed to same in the available nutrients (both in QTP). Nevertheless, previous studies demonstrated that the relative abundance of Firmicutes were higher than Bacteroidetes in yak and bison at grazing patterns (Huang et al. 2017; Noel et al. 2017). The reason related to the low Firmicutes abundance in this study might be this phylum could be impacted by numerous factors, such as age (Xufeng et al. 2015), location (Huang et al. 2017) and diet (Pitta et al. 2010). Guo et al. (2008) reported that a low-calorie diet that is rich in protein and fiber seems to favor Bacteroidetes. It is reported that artificial high-energy diets that are rich in starch and fat seem to favor Firmicutes. The same conclusions were achieved in the current study, where the content of $\mathrm{CP}$ was positively correlated with Firmicutes and had the lowest value in the WGY group. The relative abundance of Firmicutes during GY is higher than at other stages, which might be due to alpine meadow nutrients and yield reached peaks and animals quickly gained weight from July to October. Xue et al. (2005) and Ley et al. (2006) showed that Firmicutes and body weight follow a specific positive correlation in the human gut microbiota, which might be part of the reason why Firmicutes had higher relative abundance in GY and lower relative abundance in WGY. Huang et al. (2017) reported the proportions of Firmicutes in sheep of the QTP were higher compared to those of low-elevation sheep and goats and that Gram-positive bacteria may play an important role in the digestion of specific grass on the QTP. This also explained the significant changes of

364 Firmicutes in the three stages in the QTP. As in other studies of yak grazed on QTP, the three most abundant microbial phyla were Firmicutes, Bacteroidetes, and Proteobacteria (Dan et al. 2016; Huang et al. 2017; Xue et al. 2017). However, in the current study, third most abundant phylum was verrucomictobia (5.97\%), followed by Proteobacteria (1.92\%). Studies have shown that verrucomictobia contains a broad repertoire of glycoside hydrolyze enzymes and make a significant contribution to 
369

370

371

372

373

374

375

376

377

378

379

380

381

382

383

384

385

386

387

388

389

390

391

392

393

394

395

polysaccharide and cellobiose degradation (Zoetendal et al. 2003; Godoy-Vitorino et al. 2012; Gharechahi et al. 2015). In this study, the higher relative abundance of verrucomictobia compared to other study might be caused by different grassland types. Synergistetes have been detected in mammalian intestinal tracts (Shah et al. 2009). However, the function of cellulose and xylan-pectin enrichments from Synergistetes was not clear enough. It might be the one reason that Synergistetes in the rumen had the low relative abundance in the current study. The phylum Fibrobacteres was considered to cellulolytic bacteria and shown to be influenced by fiber content in the diet (Fernando et al. 2010; Jami \& Mizrahi 2012; Paz et al. 2018). Several studies of the rumen microbiome have suggested that the phylum Fibrobacteres varies considerably across cows and diets. It is reasonable that these phylum of different abundance in this paper due to different fiber content in the forage in three seasons.

At the family level, few differences were reported in the ruminal microbiota of dairy cattle fed with either grass or total mixed rations (de Menezes et al. 2011). The greatest differences were found in Lachnospiraceae and Veillonellaceae in this study. Lachnospiraceae found in Holstein cows rumen and play important role in fibrolytic bacteria (Godoy-Vitorino et al. 2012; Ziemer 2014). The same conclusion was also reached in the current study, where the relative abundances of Lachnospiraceae reached 12.62\% (REGY). de Menezes et al. (2011) also reported that Veillonellaceae were observed at levels three times higher in cows that were fed with forage grass, which corroborates the conclusion reached in this study, since these microbial families were found in large proportions in yaks that grazed on pasture. Wang et al. (2019) reported that many microbes that belong to the family of Ruminococcaceae are fibrous-degrading bacteria with the ability to degrade protein. In the current study, the lowest relative abundance of Ruminococcaceae was presented in WGY, which is consistent with the change of $\mathrm{CP}$ in three periods. Studies demonstrated that members of the Bacteroidetes phylum that contain a varied combination of CAZymes (Carbohydrate-Active Enzymes) in the form of PULs (polysaccharide utilization loci), which encode multiple proteins include the detection, sequestration, hydrolysis and transport of complex carbohydrates(Gharechahi \& Salekdeh 2018). It is reported that particular PUL can be used to predict the substrate specificity of microbial strains and the presence of many PULs in Bacteroidetes genomes indicates their broad substrate specifcity and high potential carbohydrate degradation ability (Stewart et 
396

397

398

399

400

401

402

403

404

405

406

407

408

409

410

411

412

413

414

415

416

417

418

419

420

421

422

al. 2018). Therefore, Bacteroidales_BS11_gut_group, Bacteroidales_S24-7_group, and

Bacteroidales_RF16_group all account for a large proportion in this study due to their wide functions.

The forage growth stage ( $p=0.015$, Fig. 5) and the gender of the animal (GY, $p=0.018$, Fig. 5) all had significant effects on Bacteroidales_S24-7_group in the current study and their relative abundances were highest during GY (Table S3). Research by Long et al. (1999) showed that soluble sugars are susceptible to seasonal and regional effects and reached a maximum in July in the QTP region. Stewart et al. (2018) also identify RUGs with large numbers of PUL related to Prevotellaceae family that contain proteins capable of binding and digesting multiple carbohydrate substrates. Consistent with this, the large relative abundance of Prevotellaceae in the rumen of yak in this study indicated that these family related to the high potential carbohydrate degradation ability. Two recent studies reported that Christensenellaceae, Ruminococcaceae, Rikenellaceae, and Prevotellaceae may play important roles in forage degradation in the rumen since these groups tightly adhere to forage grass after incubation in the rumen (Liu et al. 2016; Shen et al. 2017). In addition, it has been reported that the abundance of Christensenellaceae is associated with changes in rumen $\mathrm{pH}$ (De et al. 2016).

At the genus level, bacteria of Treponema in the rumen are able to degrade plant polysaccharides from hay or from a concentrated diet. As one of the core members of the rumen bacterial community (Bekele et al. 2011), the same conclusion could also be reached in the current study, where the Treponema_2 showed similar relative abundance during all three forage growth stages, with an average abundance of $0.7 \%$. The genus Butyrivibrio plays an important role in the decomposition of urea, protein, hemicellulose, cellulose, and complex carbohydrates (Peng et al. 2015). In this study, the relative abundance of Butyrivibrio_2 in the GY was significantly higher $(\mathrm{P}<0.05)$ than in the other two periods. Prevotella is capable to metabolize dietary fibers from plant cell walls, and thus produces significant amounts of short chain fatty acids (SCFAs) that are later absorbed by the animals (Ramayo-Caldas et al. 2016). Furthermore, Prevotella, which participates in the processing of complex dietary polysaccharides (Ellekilde et al. 2014), may promote an increased uptake of monosaccharides in the host. The average abundance of Prevotella reached $15.62 \%$ in the current study. Prevotella participates in the degradation of cellulose to produce VFA and break down polysaccharides in the plant into monosaccharides, thus 
423

424

425

426

427

428

429

430

431

432

433

434

435

436

437

438

439

440

441

442

443

444

445

446

447

448

449

supplying the yak with energy. This results in its higher relative abundance during WGY due to the increased fiber and lignin contents as well as the low nutrient content in the alpine meadow. Consequently, yaks must increase their feed intake to fight cold weather during winter. Prevotella is a large genus with high species diversity and plays an important role in the degradation of cellulose and vegetable protein (Ley et al. 2006). Clostridium is responsible for the degradation of cellulose (Burrell et al. 2004) and is commonly found when studying yaks. However, in this study, the relative abundance of Clostridium was $<0.05 \%$, which may be due to the degradation of cellulose in yaks by other and currently undiscovered fiber-degrading bacteria or by the nutrients related to the forage grass (Bergmann et al. 2015). The current study reached the same conclusion as the previous publication by (Shen et al., 2017), which showed that the abundance of fibrolytic bacterial genera (e.g., Ruminococcus and Butyrivibrio) and potentially fibrolytic bacterial taxa (e.g. Christensenellaceae- $R-7$, unclassified Ruminococcaceae, and unclassified Rikenellaceae) all occupy a majority at the genus level.

Bolnick et al. (2014) have shown that when male and female rats are fed the same high-fat diet, they show changes in relative abundance of gut bacteria only at the species level. When there is a difference in diet, males and females show apparent differences. In the current study, no difference was applied in the diets between male and female yaks during the same period. Consequently, under the same dietary conditions, male and female yaks only showed differences in relative abundance during REGY, GY, and WGY (Fig. 5). Abreu (2010) reported that Toll-like receptors (TLR) as a receptors which can mediating innate immune responses, and related microbiota. Liu et al. (2017) showed that newborn lambs with concentrates starter feeding increased the colonic mucosal mRNA expression of TLR and expression of TLR was associated with changes in the abundances of some specific bacteria like unclassified S24-7. Trevisi et al. (2014) indicated that bovine rumen can received the immune cells. The Bacteroidales_S24-7 group was higher in female yak than male yak during GY, the newborn animals intestinal flora were more closely associated with maternal, so the high Bacteroidales_S24-7_group in female yaks in this harsh environment may be related to this but it has not been confirmed. In male yaks, the relative abundance of phylum Bacteroidetes (WGY), family Prevotellaceae (WGY), and Prevotella_l (WGY) was significantly higher $(\mathrm{P}<0.05)$ than in female yaks. In the current study, gender factors make it 
450

451

452

453

454

455

456

457

458

459

460

461

462

463

464

465

466

467

468

469

470

471

472

473

474

475

476

difficult to interpret these results, but when the diet is compared to other factors, the gender of the animal exerts a relatively small effect on the composition of the microbiota (Bergmann et al. 2015; Bergmann 2017).

Microorganisms influence immune function, nutrient absorption, and even enzyme metabolism (Martin et al. 2010). Tax4Fun is a software package that predicts the functional capabilities of microbial communities based on 16S rRNA datasets and Tax4Fun provides good functional profiles obtained from metagenomic shotgun sequencing approaches (Asshauer et al. 2015). Nucleotide excision, RNA degradation, Peptidoglycan biosynthesis, Starch and sucrose metabolism, and Amino sugar and nucleotide sugar metabolism were the most abundant functional genes in this study. KOs related to ABC transporters were reported as the the largest known protein family and are ubiquitously found in bacteria, archaea, and eukaryotes (Zeng et al. 2017). It might be the one reason that these KOs could found in high abundance in the rumen microbiota in this study. Besides, Guo et al. (2018) have shown that pathway related to ABC transporters was enriched on yaks and sheep grazing in the QTP. Hamana et al. (2012) demonstrated that $\mathrm{ABC}$ transport function as a barrier to protect the bovine not to be hurt from the intake of toxic substances. It is reasonable that these KOs of high abundance due to yak's ability to adapt to bad weather led to the increased metabolic function of the $\mathrm{ABC}$ transporters. Two-component systems are perceptually developed signal transduction systems (Podgornaia \& Laub 2013; Zeng et al. 2017). In the current study, KOs include ABC transporters, starch and Sucrose metabolism, and Amino sugar and nucleotide sugar metabolism, all of which had high relatively abundance during REGY, which might be related to the high CP content at the alpine meadow during REGY. The results of level 2 KOs (Table S5) suggest that the pathways related to Membrane transport had high abundance, which might be related to the high altitude and cold climate in the QTP, because the nutritional composition of alpine meadow grass differs, which in turn affects both the diversity and function of the bacteria in the yak rumen. According to the heatmap of $20 \mathrm{KOs}$ in the level $3 \mathrm{KEGG}$ pathway (Fig. S2), the functional KOs of rumen microbiota varied considerably in REGY, GY, and WGY.

\section{Conclusions}


This study reports the effect of the forage on rumen microbiol diversity in yak of the QTP. Different forage growth stages changed the composition, diversity, and function of rumen microbiota in yaks that grazed naturally without feed supplementation in the alpine meadow of the QTP. Changes in temperature directly affect plant productivity, which in turn affects animal microbiome in the body. This effect is not related to the gender of the yaks. More studies about fungal amplicon sequencing (ITS) are needed to explore and the relationship between the microbial changes at different forage growth stages and gender could be better illustrated. Furthermore, research about archaeal composition in the rumen of yaks are required to promote the understanding of the reduction of $\mathrm{CH} 4$ emission and can also enhance the livestock production efficiency.

\section{Acknowledgements}

The authors would like to acknowledge the warm-hearted help of the research group during the sample collection and experimental data analysis.

\section{REFERENCE}

Abreu MT. 2010. Toll-like receptor signalling in the intestinal epithelium: how bacterial recognition shapes intestinal function. Nature Reviews Immunology 10:131-144.

Asshauer KP, Wemheuer B, Daniel R, and Meinicke P. 2015. Tax4Fun: predicting functional profiles from metagenomic 16S rRNA data. Bioinformatics 31:2882-2884. 10.1093/bioinformatics/btv287

Bekele AZ, Koike S, and Kobayashi Y. 2011. Phylogenetic diversity and dietary association of rumen Treponema revealed using group-specific 16S rRNA gene-based analysis. FEMS Microbiol Lett 316:51-60. 10.1111/j.1574-6968.2010.02191.x

Belanche A, Doreau M, Edwards JE, Moorby JM, Pinloche E, and Newbold CJ. 2012. Shifts in the rumen microbiota due to the type of carbohydrate and level of protein ingested by dairy cattle are associated with changes in rumen fermentation. $J$ Nutr 142:1684-1692. 10.3945/jn.112.159574

Bergmann GT. 2017. Microbial community composition along the digestive tract in forage- and grain-fed bison. BMC Vet Res 13:253. 10.1186/s12917-017-1161-x

Bergmann GT, Craine JM, Robeson MS, 2nd, and Fierer N. 2015. Seasonal Shifts in Diet and Gut Microbiota of the American Bison (Bison bison). PLoS One 10:e0142409. 10.1371/journal.pone.0142409

Bolnick DI, Snowberg LK, Hirsch PE, Lauber CL, Org E, Parks B, Lusis AJ, Knight R, Caporaso JG, and Svanback R. 2014. Individual diet has sex-dependent effects on vertebrate gut microbiota. Nat Commun 5:4500. 10.1038/ncomms5500

Burrell PC, O'Sullivan C, Song H, Clarke WP, and Blackall LL. 2004. Identification, detection, and spatial resolution of Clostridium populations responsible for cellulose degradation in a methanogenic landfill 
leachate bioreactor. Appl Environ Microbiol 70:2414-2419.

Caporaso JG, Kuczynski J, Stombaugh J, Bittinger K, Bushman FD, Costello EK, Fierer N, Pena AG, Goodrich JK, Gordon JI, Huttley GA, Kelley ST, Knights D, Koenig JE, Ley RE, Lozupone CA, McDonald D, Muegge BD, Pirrung M, Reeder J, Sevinsky JR, Turnbaugh PJ, Walters WA, Widmann J, Yatsunenko T, Zaneveld J, and Knight R. 2010. QIIME allows analysis of high-throughput community sequencing data. Nat Methods 7:335-336. 10.1038/nmeth.f.303

CHEN, X. L, WANG, J. K, WU, Y. M, LIU, and J. X. 2008. Effects of chemical treatments of rice straw on rumen fermentation characteristics, fibrolytic enzyme activities and populations of liquid-and solid-associated ruminal microbes in vitro. Animal Feed Science \& Technology 141:1-14.

Chen YB, Fu M, Lan DL, and Li J. 2015a. Molecular Characterization and Expression Analysis of Insulin-like Growth Factor-1 and Insulin-like Growth Factor Binding Protein-1 Genes in Qinghai-Tibet Plateau Bos grunniens and Lowland Bos taurus. Asian-Australas J Anim Sci 28:20-24. 10.5713/ajas.14.0413

Chen YB, Lan DL, Tang C, Yang XN, and Li J. 2015b. Effect of DNA Extraction Methods on the Apparent Structure of Yak Rumen Microbial Communities as Revealed by 16S rDNA Sequencing. Pol J Microbiol 64:29-36.

Cunniff P. 1995. Official Methods of Analysis of AOAC International. Aoac Official Method 6:382-382.

Dan X, Chen H, Chen F, He Y, Zhao C, Zhu D, Zeng L, and Li W. 2016. Analysis of the rumen bacteria and methanogenic archaea of yak (Bos grunniens) steers grazing on the Qinghai-Tibetan Plateau. Livestock Science 188:61-71. 10.1016/j.livsci.2016.04.009

de Menezes AB, Lewis E, O'Donovan M, O'Neill BF, Clipson N, and Doyle EM. 2011. Microbiome analysis of dairy cows fed pasture or total mixed ration diets. FEMS Microbiol Ecol 78:256-265. 10.1111/j.15746941.2011.01151.x

De NR, Marchesini G, Li S, Khafipour E, Plaizier KJ, Gianesella M, Ricci R, Andrighetto I, and Segato S. 2016. Metagenomic analysis of rumen microbial population in dairy heifers fed a high grain diet supplemented with dicarboxylic acids or polyphenols. Bmc Veterinary Research 12:29.

Dehority BA, Tirabasso PA, and Grifo AP. 1989. Most-probable-number procedures for enumerating ruminal bacteria, including the simultaneous estimation of total and cellulolytic numbers in one medium. Applied \& Environmental Microbiology 55:2789-2792.

Desantis TZ, Hugenholtz P, Larsen N, Rojas M, Brodie EL, Keller K, Huber T, Dalevi D, Hu P, and Andersen GL. 2006. Greengenes: Chimera-checked 16S rRNA gene database and workbenchcompatible in ARB. Applied \& Environmental Microbiology 72:5069-5072.

Edgar RC. 2013. UPARSE: highly accurate OTU sequences from microbial amplicon reads. Nat Methods 10:996998. 10.1038/nmeth.2604

Ellekilde M, Krych L, Hansen CH, Hufeldt MR, Dahl K, Hansen LH, Sorensen SJ, Vogensen FK, Nielsen DS, and Hansen AK. 2014. Characterization of the gut microbiota in leptin deficient obese mice - Correlation to inflammatory and diabetic parameters. Res Vet Sci 96:241-250. 10.1016/j.rvsc.2014.01.007

Fernandes KA, Kittelmann S, Rogers CW, Gee EK, Bolwell CF, Bermingham EN, and Thomas DG. 2014. Faecal microbiota of forage-fed horses in New Zealand and the population dynamics of microbial communities following dietary change. PLoS One 9:e112846. 10.1371/journal.pone.0112846

Fernandez-Guerra A, Buchan A, Mou X, Casamayor EO, and Gonzalez JM. 2010. T-RFPred: a nucleotide sequence size prediction tool for microbial community description based on terminal-restriction fragment 
length polymorphism chromatograms. BMC Microbiol 10:262. 10.1186/1471-2180-10-262

Fernando SC, Purvis HT, Najar FZ, Sukharnikov LO, Krehbiel CR, Nagaraja TG, Roe BA, and Desilva U, . 2010. Rumen microbial population dynamics during adaptation to a high-grain diet. Appl Environ Microbiol 76:7482-7490.

Gharechahi J, and Salekdeh GH. 2018. A metagenomic analysis of the camel rumen's microbiome identifies the major microbes responsible for lignocellulose degradation and fermentation. Biotechnology for Biofuels 11:216. 10.1186/s13068-018-1214-9

Gharechahi J, Zahiri HS, Noghabi KA, and Salekdeh GH. 2015. In-depth diversity analysis of the bacterial community resident in the camel rumen. Syst Appl Microbiol 38:67-76. 10.1016/j.syapm.2014.09.004

Godoy-Vitorino F, Goldfarb KC, Karaoz U, Leal S, Garcia-Amado MA, Hugenholtz P, Tringe SG, Brodie EL, and Dominguez-Bello MG. 2012. Comparative analyses of foregut and hindgut bacterial communities in hoatzins and cows. ISME $J$ 6:531-541. 10.1038/ismej.2011.131

Guo M, Wu F, Hao G, Qi Q, Li R, Li N, Wei L, and Chai T. 2017. Bacillus subtilis Improves Immunity and Disease Resistance in Rabbits. Front Immunol 8:354. 10.3389/fimmu.2017.00354

Guo W, Bi S, Kang J, Zhang Y, Long R, Huang X, Shan MN, and Anderson RC. 2018. Bacterial communities related to 3-nitro-1-propionic acid degradation in the rumen of grazing ruminants in the QinghaiTibetan Plateau. Anaerobe 54:42-54. 10.1016/j.anaerobe.2018.07.013

Guo X, Xia X, Tang R, and Wang K. 2008. Real-time PCR quantification of the predominant bacterial divisions in the distal gut of Meishan and Landrace pigs. Anaerobe 14:224-228. 10.1016/j.anaerobe.2008.04.001

Hamana K, Itoh T, Sakamoto M, and Hayashi H. 2012. Covalently linked polyamines in the cell wall peptidoglycan of the anaerobes belonging to the order Selenomonadales. $J$ Gen Appl Microbiol 58:339347.

Harris RB. 2010. Rangeland degradation on the Qinghai-Tibetan plateau: A review of the evidence of its magnitude and causes. Journal of Arid Environments 74:1-12. 10.1016/j.jaridenv.2009.06.014

Huang J, Li Y, and Luo Y. 2017. Bacterial community in the rumen of Tibetan sheep and Gansu alpine finewool sheep grazing on the Qinghai-Tibetan Plateau, China. J Gen Appl Microbiol 63:122-130. 10.2323/jgam.2016.08.003

Jami E, and Mizrahi I. 2012. Composition and similarity of bovine rumen microbiota across individual animals. PLoS One 7:e33306. 10.1371/journal.pone.0033306

Klitgaard K, Foix Breto A, Boye M, and Jensen TK. 2013. Targeting the treponemal microbiome of digital dermatitis infections by high-resolution phylogenetic analyses and comparison with fluorescent in situ hybridization. J Clin Microbiol 51:2212-2219. 10.1128/JCM.00320-13

Latham EA, Weldon KK, Wickersham TA, Coverdale JA, and Pinchak WE. 2018. Responses in the rumen microbiome of Bos taurus and indicus steers fed a low-quality rice straw diet and supplemented protein. J Anim Sci 96:1032-1044. 10.1093/jas/sky023

Ley RE, Turnbaugh PJ, Klein S, and Gordon JI. 2006. Microbial ecology: human gut microbes associated with obesity. Nature 444:1022-1023. 10.1038/4441022a

Liu J, Bian G, Sun D, Zhu W, and Mao S. 2017. Starter Feeding Supplementation Alters Colonic Mucosal Bacterial Communities and Modulates Mucosal Immune Homeostasis in Newborn Lambs. Front Microbiol 8:429. 10.3389/fmicb.2017.00429

Liu J, Zhang M, Xue C, Zhu W, and Mao S. 2016. Characterization and comparison of the temporal dynamics 
of ruminal bacterial microbiota colonizing rice straw and alfalfa hay within ruminants. $J$ Dairy Sci 99:9668-9681. 10.3168/jds.2016-11398

Long RJ, Apori SO, Castro FB, and Orskov ER. 1999. Feed value of native forages of the Tibetan Plateau of China. Animal Feed Science \& Technology 80:101-113.

Martin R, Nauta AJ, Ben Amor K, Knippels LM, Knol J, and Garssen J. 2010. Early life: gut microbiota and immune development in infancy. Benef Microbes 1:367-382. 10.3920/BM2010.0027

Minoru K, Susumu G, Yoko S, Miho F, and Mao T. 2012. KEGG for integration and interpretation of large-scale molecular data sets.

Morozumi M, Nakayama E, Iwata S, Aoki Y, Hasegawa K, Kobayashi R, Chiba N, Tajima T, and Ubukata K. 2006. Simultaneous detection of pathogens in clinical samples from patients with community-acquired pneumonia by real-time PCR with pathogen-specific molecular beacon probes. J Clin Microbiol 44:14401446. 10.1128/JCM.44.4.1440-1446.2006

Noel SJ, Attwood GT, Rakonjac J, Moon CD, Waghorn GC, and Janssen PH. 2017. Seasonal changes in the digesta-adherent rumen bacterial communities of dairy cattle grazing pasture. PLoS One 12:e173819. 10.1371/journal.pone.0173819

Paz HA, Hales KE, Wells JE, Kuehn LA, Freetly HC, Berry ED, Flythe MD, Spangler ML, and Fernando SC. 2018. Rumen bacterial community structure impacts feed efficiency in beef cattle. J Anim Sci 96:1045-1058. 10.1093/jas/skx081

Peng S, Yin J, Liu X, Jia B, Chang Z, Lu H, Jiang N, and Chen Q. 2015. First insights into the microbial diversity in the omasum and reticulum of bovine using Illumina sequencing. $J$ Appl Genet 56:393-401. 10.1007/s13353-014-0258-1

Pitta DW, Pinchak E, Dowd SE, Osterstock J, Gontcharova V, Youn E, Dorton K, Yoon I, Min BR, Fulford JD, Wickersham TA, and Malinowski DP. 2010. Rumen bacterial diversity dynamics associated with changing from bermudagrass hay to grazed winter wheat diets. Microb Ecol 59:511-522. 10.1007/s00248-009-9609-6

Podgornaia AI, and Laub MT. 2013. Determinants of specificity in two-component signal transduction. Curr Opin Microbiol 16:156-162. 10.1016/j.mib.2013.01.004

Quast C, Pruesse E, Yilmaz P, Gerken J, Schweer T, Yarza P, Peplies J, and Glockner FO. 2013. The SILVA r ibosomal RNA gene database project: improved data processing and webbased tools. Nucleic Acids Research 41:590-596.

Ramayo-Caldas Y, Mach N, Lepage P, Levenez F, Denis C, Lemonnier G, Leplat JJ, Billon Y, Berri M, Dore J, Rogel-Gaillard C, and Estelle J. 2016. Phylogenetic network analysis applied to pig gut microbiota identifies an ecosystem structure linked with growth traits. ISME J 10:2973-2977. 10.1038/ismej.2016.77

Ramos-Morales E, Arco-Pérez A, Martín-García AI, Yáñez-Ruiz DR, Frutos P, and Hervás G. 2014. Use of stomach tubing as an alternative to rumen cannulation to study ruminal fermentation and microbiota in sheep and goats. Animal Feedence \& Technology 198:57-66.

Sadet-Bourgeteau S, Martin C, and Morgavi DP. 2010. Bacterial diversity dynamics in rumen epithelium of wethers fed forage and mixed concentrate forage diets. Vet Microbiol 146:98-104. 10.1016/j.vetmic.2010.04.029

Shah HN, Olsen I, Bernard K, Finegold SM, Gharbia S, and Gupta RS. 2009. Approaches to the study of the 
systematics of anaerobic, gram-negative, non-sporeforming rods: current status and perspectives. Anaerobe 15:179-194. 10.1016/j.anaerobe.2009.08.003

Shen J, Liu Z, Yu Z, and Zhu W. 2017. Monensin and Nisin Affect Rumen Fermentation and Microbiota Differently In Vitro. Front Microbiol 8:1111. 10.3389/fmicb.2017.01111

Stewart RD, Auffret MD, Warr A, Wiser AH, Press MO, Langford KW, Liachko I, Snelling TJ, Dewhurst RJ, and Walker AW. 2018. Assembly of 913 microbial genomes from metagenomic sequencing of the cow rumen. Nature Communications 9.

Sun J, Xu J, Shen Y, Wang M, Yu L, and Wang H. 2018. Effects of different dietary ratio of physically effective neutral detergent fiber and metabolizable glucose on rumen fermentation, blood metabolites and growth performance of 8 to 10-month-old heifers. Asian-Australas J Anim Sci 31:1230-1237. 10.5713/ajas.17.0885

Tajima K, Arai S, Ogata K, Nagamine T, Matsui H, Nakamura M, Aminov RI, and Benno Y. 2000. Rumen Bacterial Community Transition During Adaptation to High-grain Diet. Anaerobe 6:273-284. 10.1006/anae.2000.0353

Trevisi E, Amadori M, Riva F, Bertoni G, and Bani P. 2014. Evaluation of innate immune responses in bovine forestomachs. Res Vet Sci 96:69-78. 10.1016/j.rvsc.2013.11.011

Van Soest PJ, Robertson JB, and Lewis BA. 1991. Methods for dietary fiber, neutral detergent fiber, and nonstarch polysaccharides in relation to animal nutrition. $J$ Dairy Sci 74:3583-3597. 10.3168/jds.S00220302(91)78551-2

Wang L, Liu K, Wang Z, Bai X, Peng Q, and Jin L. 2019. Bacterial Community Diversity Associated With Different Utilization Efficiencies of Nitrogen in the Gastrointestinal Tract of Goats. Front Microbiol 10:239. 10.3389/fmicb.2019.00239

Wang Q, Garrity GM, Tiedje JM, and Cole JR. 2007. Naive Bayesian classifier for rapid assignment of rRNA sequences into the new bacterial taxonomy. Appl Environ Microbiol 73:5261-5267. 10.1128/AEM.0006207

Xue B, Zhao XQ, and Zhang YS. 2005. Seasonal changes in weight and body composition of yak grazing on alpine-meadow grassland in the Qinghai-Tibetan plateau of China. J Anim Sci 83:1908-1913. $10.2527 / 2005.8381908 \mathrm{x}$

Xue D, Chen H, Luo X, Guan J, He Y, and Zhao X. 2018. Microbial diversity in the rumen, reticulum, omasum, and abomasum of yak on a rapid fattening regime in an agro-pastoral transition zone. $J$ Microbiol. 10.1007/s12275-018-8133-0

Xue D, Chen H, Zhao X, Xu S, Hu L, Xu T, Jiang L, and Zhan W. 2017. Rumen prokaryotic communities of ruminants under different feeding paradigms on the Qinghai-Tibetan Plateau. Systematic \& Applied Microbiology 40:227.

Xufeng H, Yuxin Y, Hailong Y, Xiaolong W, Lei Q, and Yulin C. 2015. Rumen bacterial diversity of 80 to 110day-old goats using 16S rRNA sequencing. PLoS One 10:e117811.

Yang LY, Chen J, Cheng XL, Xi DM, Yang SL, Deng WD, and Mao HM. 2010. Phylogenetic analysis of 16S rRNA gene sequences reveals rumen bacterial diversity in Yaks (Bos grunniens). Mol Biol Rep 37:553562. 10.1007/s11033-009-9794-x

Zeng S, Huang Z, Hou D, Liu J, Weng S, and He J. 2017. Composition, diversity and function of intestinal microbiota in pacific white shrimp (Litopenaeus vannamei) at different culture stages. PeerJ 5:e3986. 
674

675

676

677

678

679

680

681

682

683 10.7717/peerj.3986

Ziemer CJ. 2014. Newly cultured bacteria with broad diversity isolated from eight-week continuous culture enrichments of cow feces on complex polysaccharides. Appl Environ Microbiol 80:574-585. 10.1128/AEM.03016-13

Zoetendal EG, Plugge CM, Akkermans ADL, and Vos WMD. 2003. Victivallis vadensis gen. nov., sp. nov., a sugar-fermenting anaerobe from human faeces. International Journal of Systematic \& Evolutionary Microbiology 53:211-215. 
Figure 1

Venn data showing shared OTUs among different forage growth stage and unique to each of them.

Only OTUs at phylum are shown.

\begin{tabular}{|c|c|}
\hline $\mathrm{F}$ & \\
\hline Spirochaetae & $(0.26 \%)$ \\
\hline Proteobacteria & $(0.69 \%)$ \\
\hline Verrucomicrobia & $(1.08 \%)$ \\
\hline Firmicutes & (3.29\%) \\
\hline Bacteroidetes & (4.87\%) \\
\hline G & \\
\hline Spirochaetae & $(0.21 \%)$ \\
\hline Verrucomicrobia & $(0.41 \%)$ \\
\hline Proteobacteria & $(0.53 \%)$ \\
\hline Firmicutes & (3.17\%) \\
\hline Bacteroidetes & $(4.99 \%)$ \\
\hline A & \\
\hline Spirochaetae & $(0.22 \%)$ \\
\hline Verrucomicrobia & $(0.33 \%)$ \\
\hline Proteobacteria & $(0.44 \%)$ \\
\hline Firmicutes & (1.20\%) \\
\hline Bacteroidetes & $(2.60 \%)$ \\
\hline
\end{tabular}

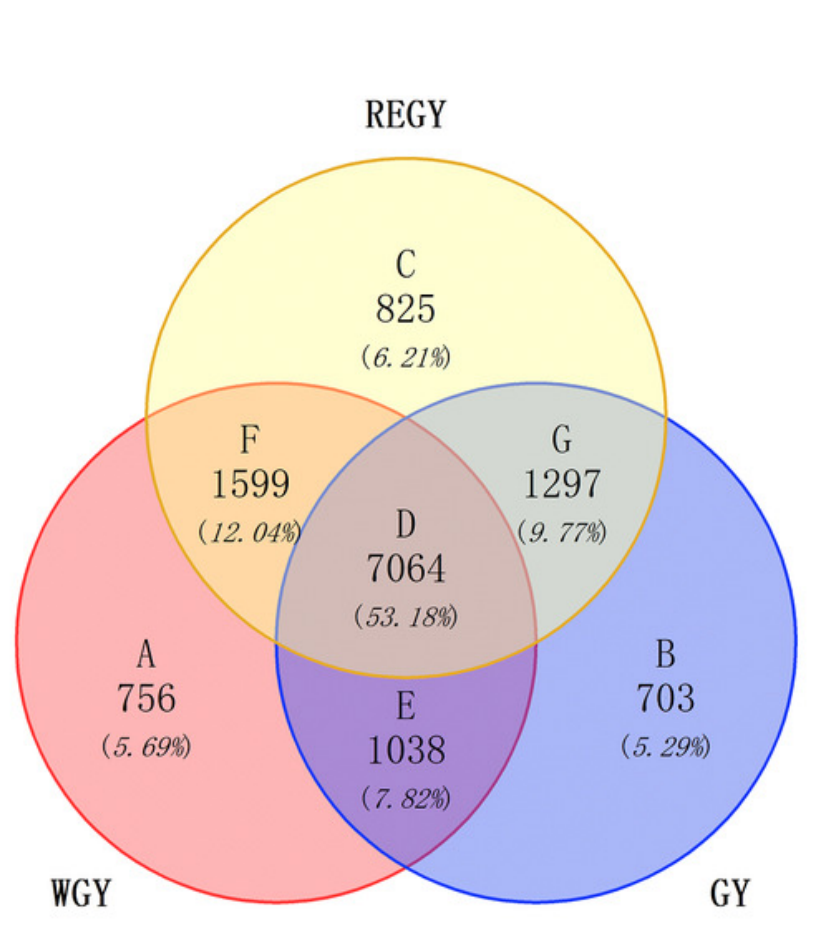

\begin{tabular}{ll}
\multicolumn{2}{c}{ D } \\
Fusobacteria & $(0.52 \%)$ \\
Cyanobacteria & $(0.63 \%)$ \\
Tenericutes & $(1.30 \%)$ \\
Spirochaetae & $(1.41 \%)$ \\
Proteobacteria & $(2.44 \%)$ \\
Verrucomicrobia & $(3.28 \%)$ \\
Firmicutes & $(17.90 \%)$ \\
Bacteroidetes & $(23.56 \%)$
\end{tabular}

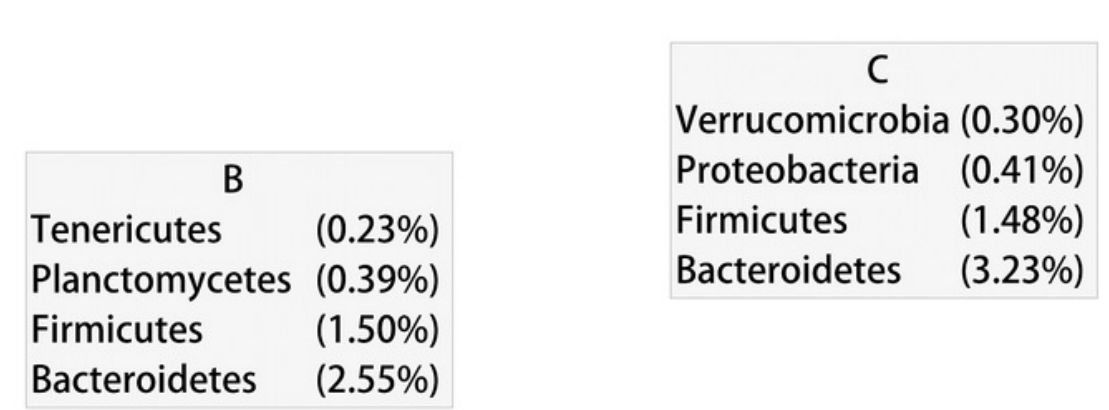




\section{Figure 2}

Differences of the relative abundance of dominant bacteria at phylum level in different grass growth stages(REGY GY and WGY).

The abundance of the top 10 phyla at different grass growth stages. The significant difference in groups by Kruskal-Wallis test with $p<0.05$ marked, the extremely significant difference in groups by Kruskal-Wallis test with $p<0.01$ marked. Student's t-test was used to compare the abundance changes each two groups (REGY vs GY, REGY vs WGY, GY and WGY). The one-asterisk above the column represents that there is significant difference in between groups ( $P$ value $<0.05$ ). 


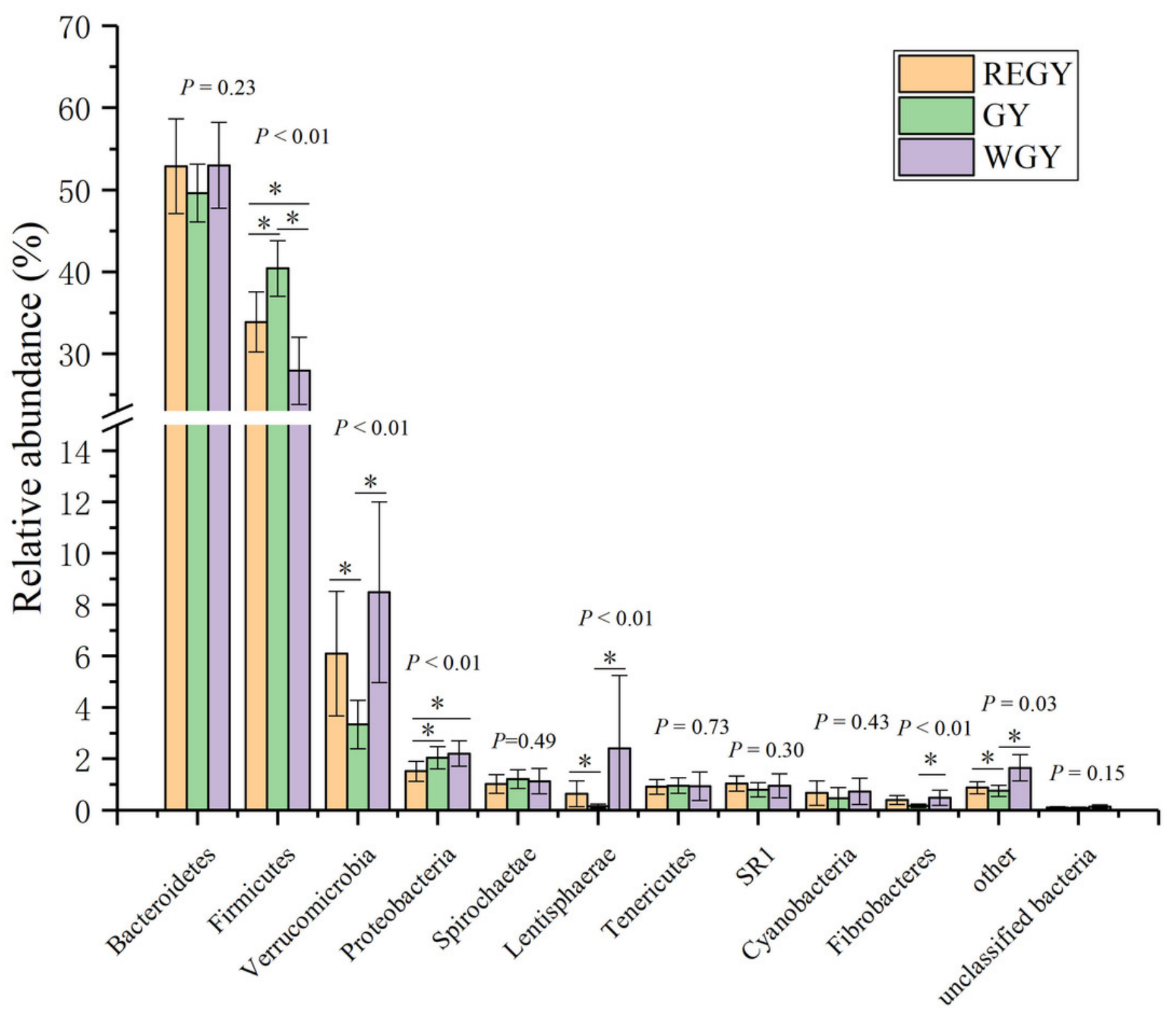


Figure 3

Differences of the relative abundance of dominant bacteria at family level in different grass growth stages(REGY GY and WGY).

The abundance of the top 10 families at different grass growth stages.

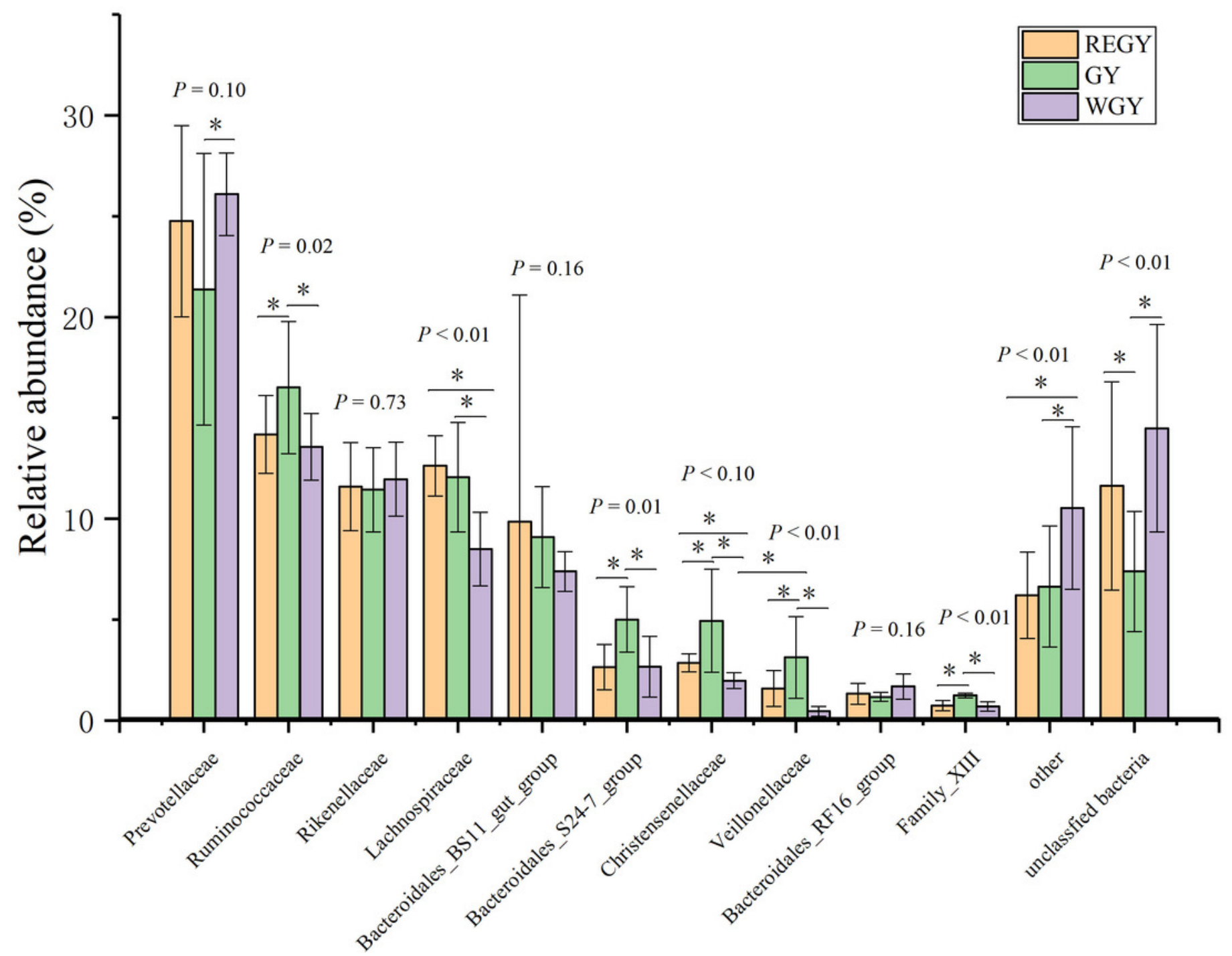


Figure 4

Differences of the relative abundance of dominant bacteria at genus level in different grass growth stages(REGY GY and WGY).

The genera of the top 10 at different grass growth stages. 


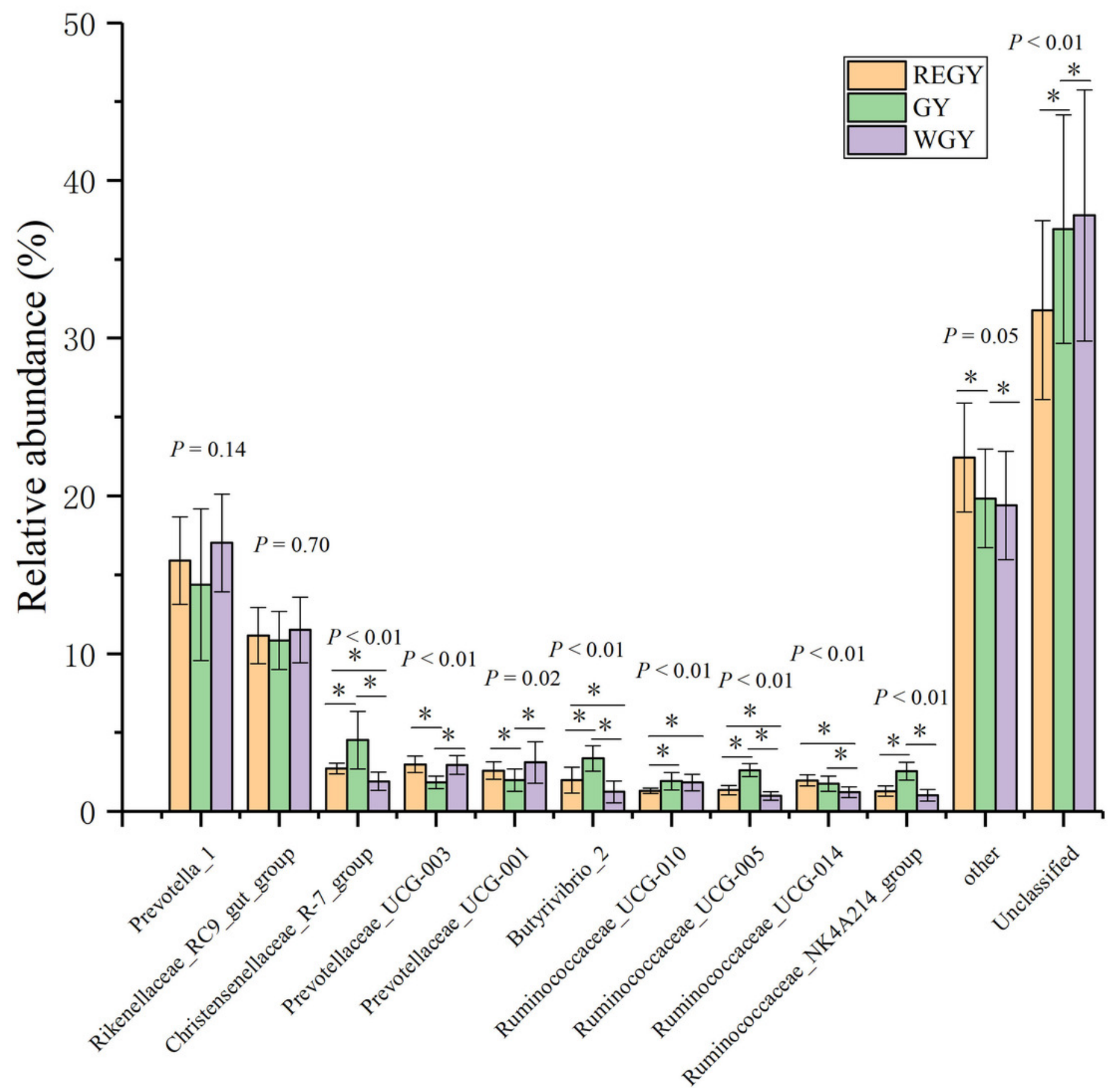


Figure 5

Percent composition and significance of phyla (A), families (B) and genera (C) between female and male yak among different grass growth stages (REGY GY and WGY).

Student's t-test was used to compare the abundance changes between female and male yaks. Only differences for which $p$-value $<0.05$ are reported. 


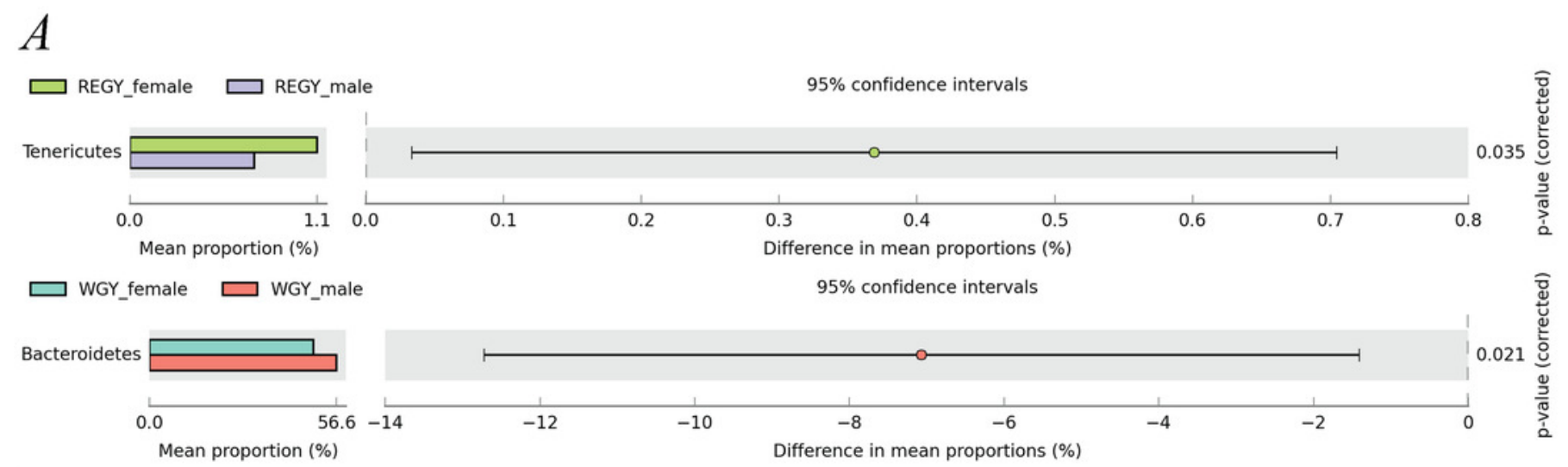

B

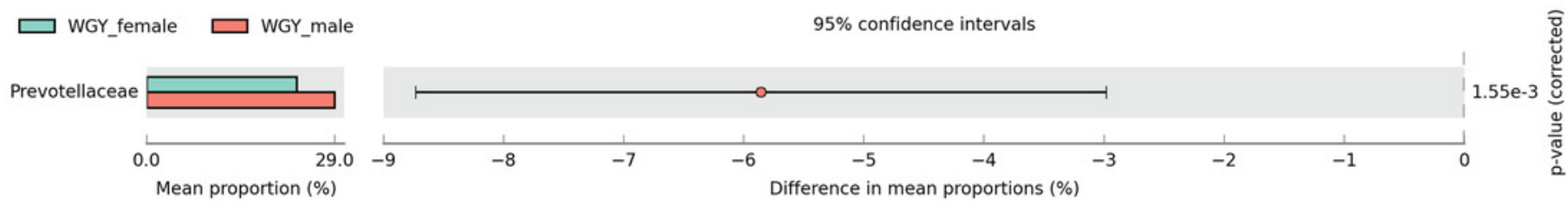

GY_female $\square$ GY_male

$95 \%$ confidence intervals

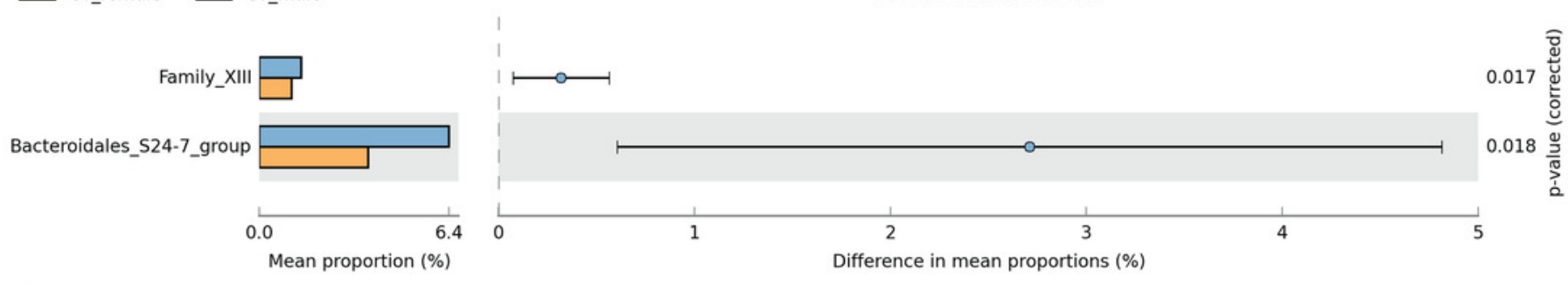

C

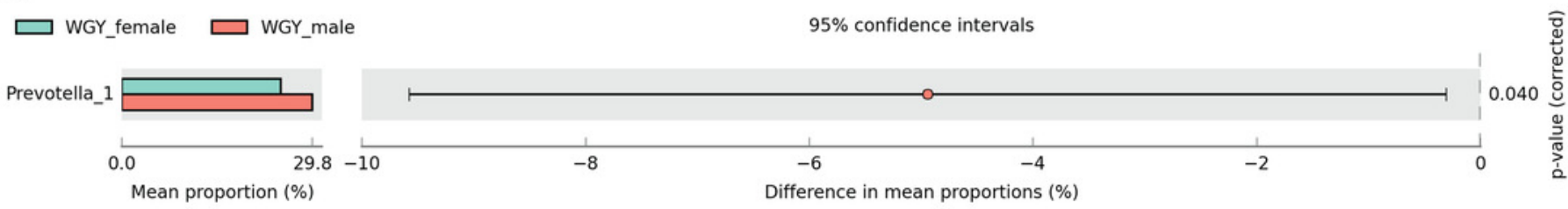

$\square$ GY_female $\square$ GY_male

Rikenellaceae_RC9_gut_group
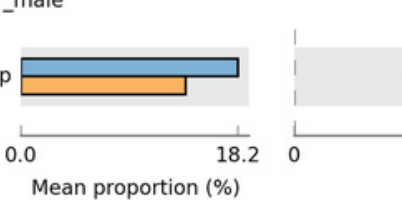

$95 \%$ confidence intervals 


\section{Figure 6}

Principal coordinate analysis ( $\mathrm{PCOA}$ ) of bacterial community structures of the ruminal microbiota among different grass growth stages.

PCoA plots were constructed using the unweighted UniFrac method, the non-parametric permutational MANOVA-based statistical tests adonis and ANOSIM. The REGY (yellow cross stands female yak ,yellow triangles stands male yak ), GY (green diamond stands female yak ,green star stands male yak) and WGY(blue cross stands female yak, blue squre stands male yak) group. 


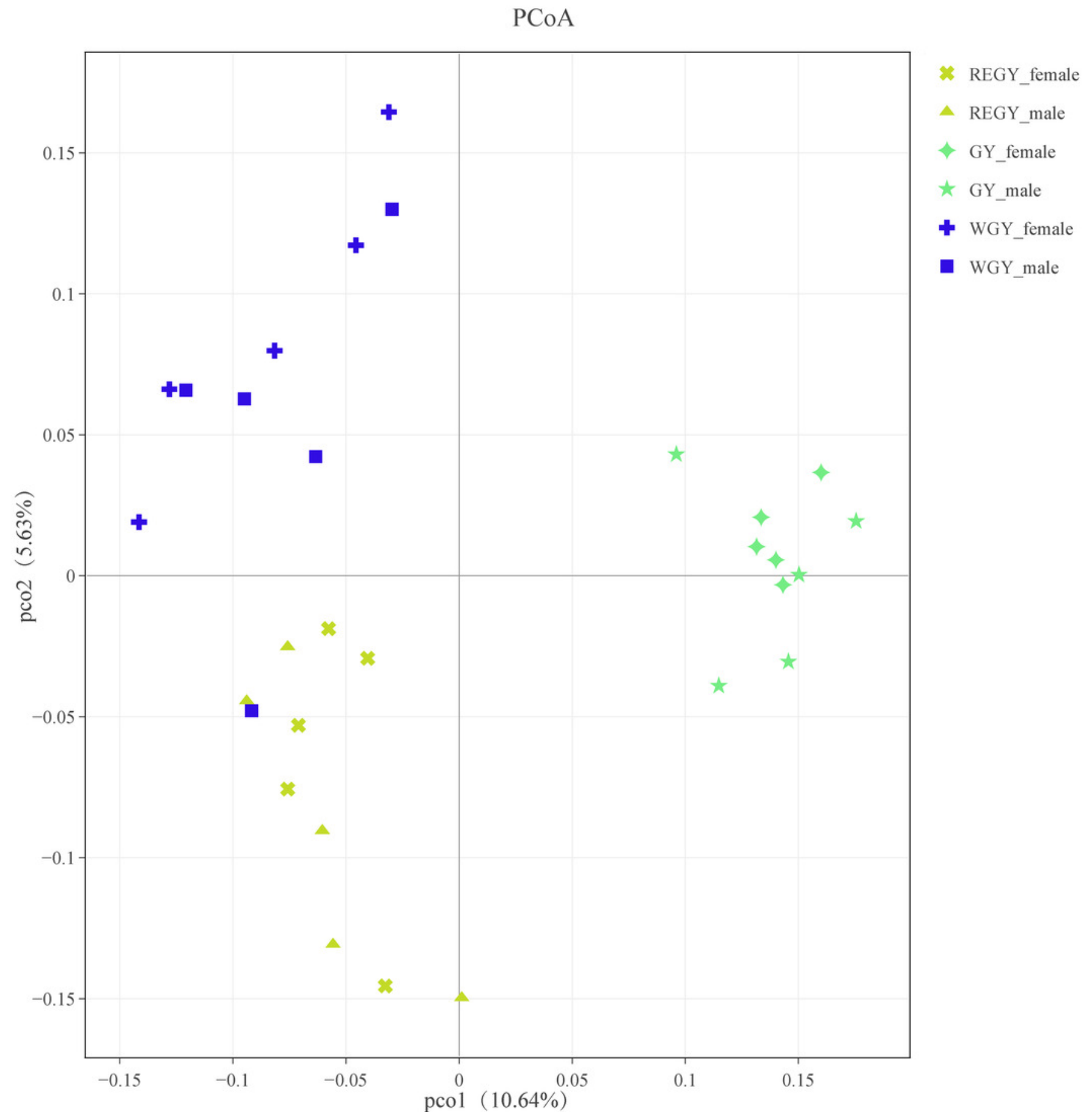




\section{Figure 7}

Correlations analyses between the relative abundances of bacteria genera and forage nutrient composition parameters.

(A) Pearson correlations analyses using the pheatmap package, the result numerical matrix is visually displayed in a heatmap diagram. The color depth indicates the size of the data value (correlation value). Red indicates that the correlation coefficients $(r)$ were $>0.4$ and the $P$ values were $<0.05$, blue indicates that the correlation coefficients $(r)$ were $<-0.4$ and the $P$ values were $<0.05$. (B) Redundancy analysis of the top 10 known prokaryotic phyla in association with 6 forage nutrient composition parameters.
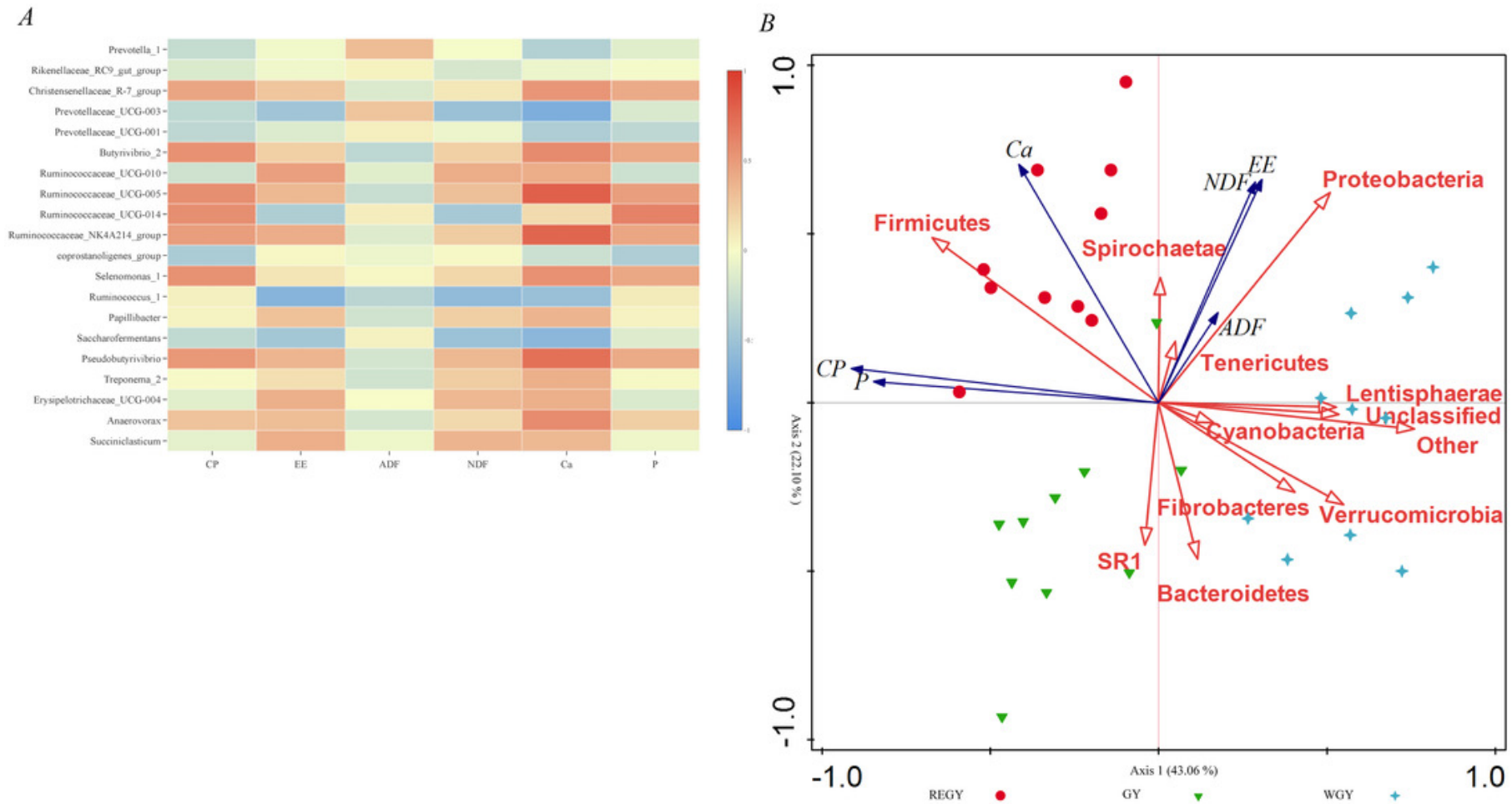
Figure 8

Relative abundance of predicted functions and significance of KOs in KEGG level 2 among three grass growth stage (REGY GY and WGY).

A (REGY vs GY); B( REGY vs WGY); C (GY and WGY). Student's t-test was used to compare the abundance changes each two groups, only differences for which $p$-value $<0.05$ are reported. 
A

$\square \mathrm{g} \square \mathrm{reg}$

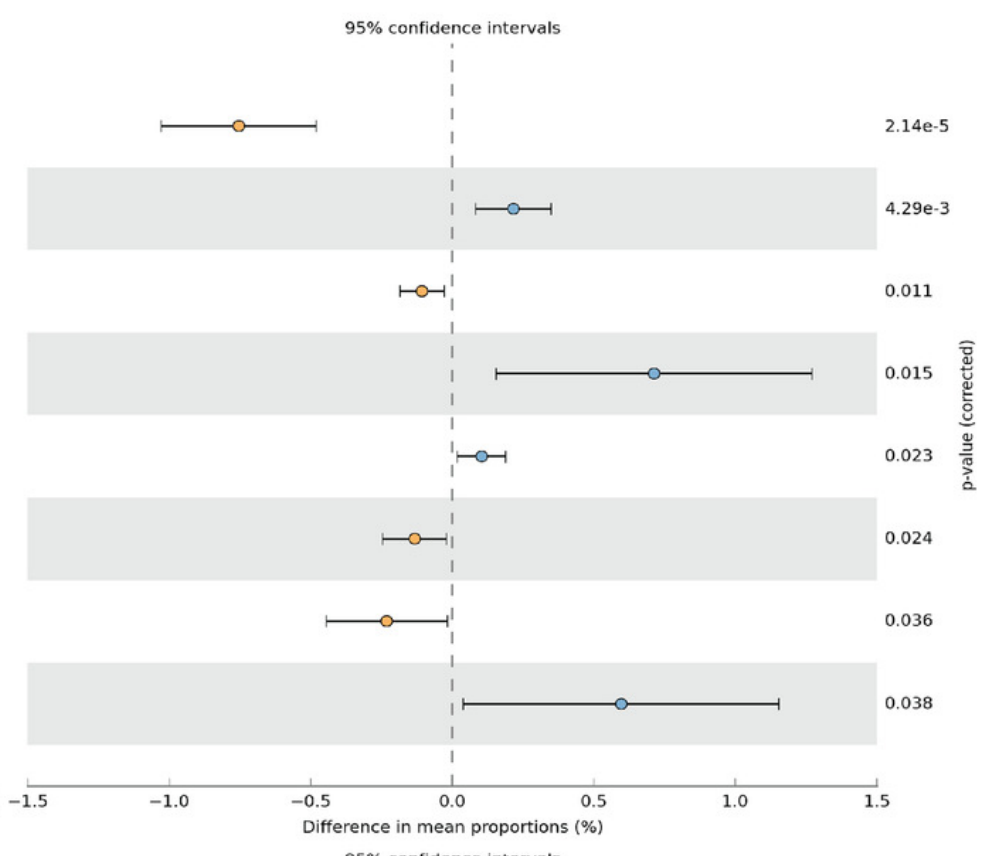

$B$

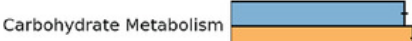

Metabolism of Cofactors and Vitamins $\square$

Glycan Biosynthesis and Metabolism

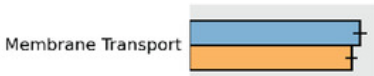

Amino Acid Metabolism

" Folding, Sorting and Degradation"

Nucleotide Metabolism

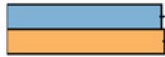

Signal Transduction

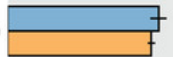

ए
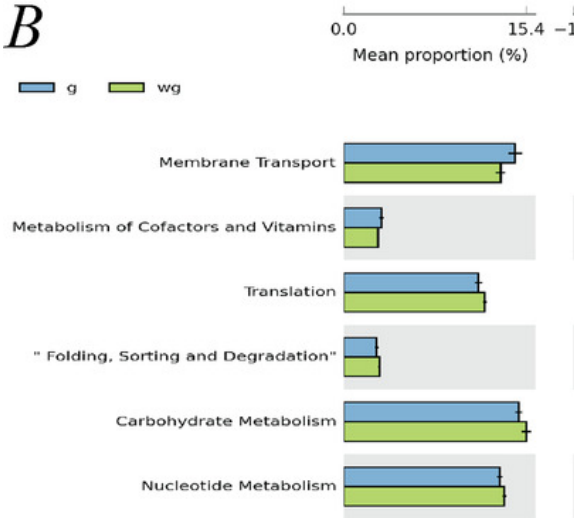

$95 \%$ confidence intervals

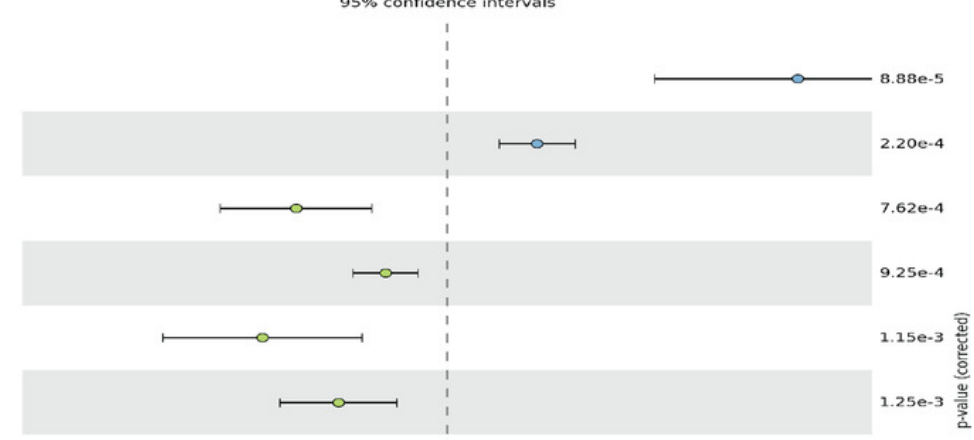

Glycan Biosynthesis and Metabolism $\square$

Cell Growth and Death $\square$

Signal Transduction

Replication and Repair

C

$\square$ reg $\square$ wg
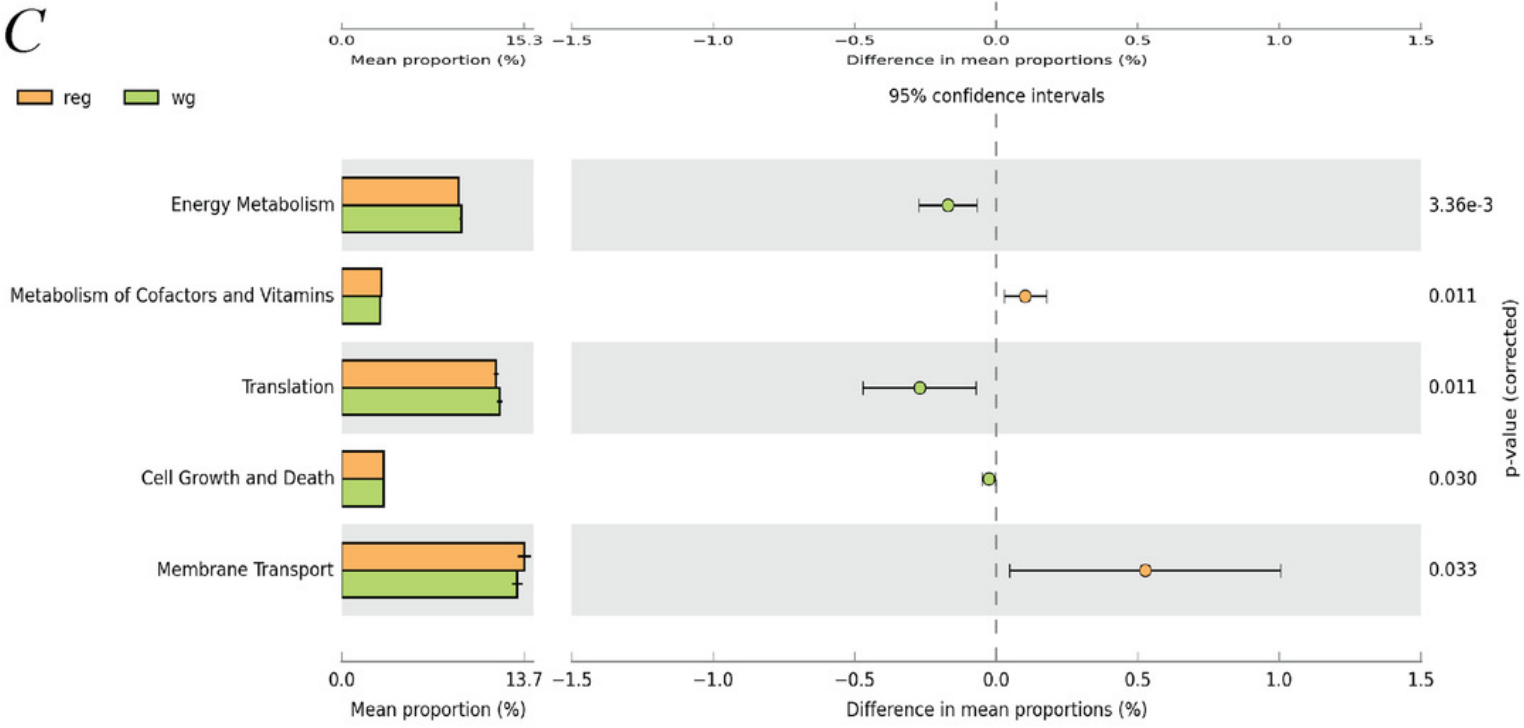

Peer] reviewing PDF | (2018:11:32825:3:0:ACCEPTED 5 Aug 2019) 
Figure 9

\section{Functional predictions for rumen microbiota that significantly different KEGG (Kyoto Encyclopedia of Genes and Genomes) pathways $(p<0.05)$ of three grass growth stage (REGY GY and WGY).}

KEGG pathway at Level 1, Level 2 and Level 3 were represented. REGY: regreen grass stage; GY: grassy grass stage; WGY: withered grass stage.

\section{level $1 \quad$ level $2 \quad$ level 3}

Metabolism $\left[\begin{array}{l}\text { Amino Acid Metabolism } \\ \text { Glycan Biosynthesis and Metabolism } \\ \text { Energy Metabolism } \\ \text { Carbonhydrate Metabolism } \quad\left[\begin{array}{r}\text { Phenylalanine, tyrosine and tryptophan biosynthesis } \\ \text { Glycine, serine and threonine metabolism } \\ \text { Cysteine and methionine metabolism }\end{array}\right. \\ \begin{array}{r}\text { Peptidoglycan biosynthesis } \\ \text { Oxidative phosphorylation } \\ \text { Nitrogen metabolism }\end{array} \\ \text { Amino sugar and nucleotide sugar metabolism } \\ \text { Starch and sucrose metabolism }\end{array}\right.$

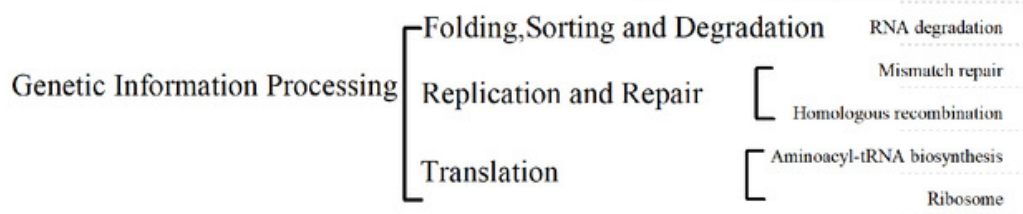
Environment Information Processing Membrane Transport ${ }^{\text {Bacterial scerction systcm }}$ Cellular Processes $\quad\left[\begin{array}{ll}\text { Cell Motility } & \begin{array}{l}\text { ABC transporters } \\ \text { Bacterial chemotaxis }\end{array} \\ \text { Cell Growth and Death Cell cycle-Caulobacter }\end{array}\right.$

$\begin{array}{lllllll}0.02 & 0.00 & 0.02 & 0.04 & 0.06 & 0.08 & 0.10 \\ \text { Relative abundance }(\%) & & \end{array}$




\section{Table 1 (on next page)}

Analysis of nutrient composition and dominant species of grassland in three grazing stages of alpine meadow in QTP

The figures in the table show the nutrient composition and main dominant species of forage at different grass growth stages.All data are mean \pm SD, the REGY refers to the re-green grass growth stage; GY refers to the grass growth stage; WGY refers to the withered grass growth stage. 


\begin{tabular}{|c|c|c|c|c|c|}
\hline \multirow{2}{*}{$\begin{array}{l}\text { Nutrient content of } \\
\text { forage grass } \%\end{array}$} & \multicolumn{3}{|c|}{ Group } & \multirow[b]{2}{*}{ SEM } & \multirow[b]{2}{*}{$p$-value } \\
\hline & REGY & GY & WGY & & \\
\hline $\mathrm{DM}$ & $93.32 \pm 0.12$ & $93.00 \pm 0.55$ & $93.10 \pm 0.48$ & 0.34 & $>0.05$ \\
\hline $\mathrm{CP}$ & $13.22 \pm 1.45 \mathrm{a}$ & $12.20 \pm 0.24 \mathrm{a}$ & $4.74 \pm 0.87 b$ & 0.14 & $<0.01$ \\
\hline $\mathrm{EE}$ & $1.07 \pm 0.08 \mathrm{~b}$ & $1.50 \pm 0.04 \mathrm{a}$ & $1.44 \pm 0.05 \mathrm{a}$ & 0.00 & $<0.01$ \\
\hline $\mathrm{ADF}$ & $35.53 \pm 1.29 \mathrm{a}$ & $30.23 \pm 1.37 \mathrm{~b}$ & $33.97 \pm 1.00 \mathrm{a}$ & 1.51 & $<0.01$ \\
\hline NDF & $52.40 \pm 3.50 \mathrm{~b}$ & $57.83 \pm 2.64 \mathrm{ab}$ & $59.00 \pm 2.43 \mathrm{a}$ & 8.37 & 0.07 \\
\hline $\mathrm{Ca}$ & $1.28 \pm 0.05 b$ & $2.18 \pm 0.12 \mathrm{a}$ & $1.27 \pm 0.08 b$ & 0.09 & $<0.03$ \\
\hline $\mathrm{P}$ & $0.12 \pm 0.02 \mathrm{a}$ & $0.14 \pm 0.00 \mathrm{a}$ & $0.04 \pm 0.02 b$ & 0.01 & $<0.04$ \\
\hline $\begin{array}{l}\text { The dominant } \\
\text { species of alpine } \\
\text { meadow in Qinghai- } \\
\text { Tibet plateau }\end{array}$ & $\begin{array}{l}\text { Mainly for those } \\
\text { Gramineae, } \\
\text { Cyperaceae and } \\
\text { Potentilla plants }\end{array}$ & $\begin{array}{c}\text { Dominant grass } \\
\text { species are Elymus } \\
\text { nutans,Poa } \\
\text { pratensi,Kobresia } \\
\text { humili etal, the } \\
\text { companion grass } \\
\text { species are } \\
\text { Potentill } \\
\text { abifurca,Saussurea } \\
\text { pulchra,Ajaniatenu } \\
\text { ifolia etal }\end{array}$ & $\begin{array}{c}\text { Dominant } \\
\text { grass species } \\
\text { are Elymus } \\
\text { nutans }\end{array}$ & & \\
\hline
\end{tabular}




\section{Table 2 (on next page)}

Rumen microbiota diversity and richness between different grass growth stages and gender.

The data in the table indicates the effects of different grass growth stages and gender on the rumen micobiota diversity and richness index. 


\begin{tabular}{lllrrrl}
\hline Grass Stage & Gender & Sobs & Simpson & Shannon & Chao & \multicolumn{1}{l}{ Ace } \\
\hline \multirow{2}{*}{ REGY } & Female & 4657.63 & 1.00 & 10.03 & 6392.91 & 6232.83 \\
\hline & Male & 4609.61 & 0.99 & 9.77 & 6419.18 & 6218.95 \\
GY & Female & 4489.22 & 1.00 & 9.84 & 6201.51 & 6089.73 \\
& Male & 4074.60 & 0.99 & 9.48 & 5712.25 & 5604.42 \\
\hline & Female & 4512.41 & 1.00 & 9.97 & 6157.27 & 5974.50 \\
WGY & Male & 4340.22 & 1.00 & 9.94 & 5900.43 & 5754.64 \\
\hline & SEM & 121.24 & 0.004 & 0.198 & 144.13 & 135.027 \\
\hline & & $p-$ value \\
\hline Grass Stage & 0.03 & 0.48 & 0.31 & 0.01 & 0.02 \\
Gender & 0.04 & 0.33 & 0.19 & 0.05 & 0.04 \\
Grass Stage $\times$ Gender & $\square$ & 0.32 & 0.56 & 0.69 & 0.22 & 0.24 \\
\hline
\end{tabular}

1 